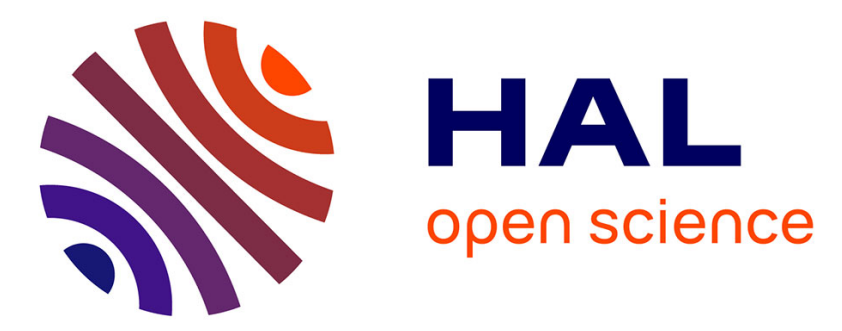

\title{
Dissipativity-based Framework for Stability Analysis of Aperiodically Sampled Nonlinear Systems with Time-varying Delay
}

Jijju Thomas, Christophe Fiter, Laurentiu Hetel, Nathan van de Wouw, Jean-Pierre Richard

\section{To cite this version:}

Jijju Thomas, Christophe Fiter, Laurentiu Hetel, Nathan van de Wouw, Jean-Pierre Richard. Dissipativity-based Framework for Stability Analysis of Aperiodically Sampled Nonlinear Systems with Time-varying Delay. Automatica, In press, 129, 10.1016/j.automatica.2021.109632 . hal-03156571

\section{HAL Id: hal-03156571 \\ https://inria.hal.science/hal-03156571}

Submitted on 2 Mar 2021

HAL is a multi-disciplinary open access archive for the deposit and dissemination of scientific research documents, whether they are published or not. The documents may come from teaching and research institutions in France or abroad, or from public or private research centers.
L'archive ouverte pluridisciplinaire HAL, est destinée au dépôt et à la diffusion de documents scientifiques de niveau recherche, publiés ou non, émanant des établissements d'enseignement et de recherche français ou étrangers, des laboratoires publics ou privés. 


\title{
Dissipativity-based Framework for Stability Analysis of Aperiodically Sampled Nonlinear Systems with Time-varying Delay *
}

\author{
Jijju Thomas ${ }^{\mathrm{a}, \mathrm{b}}$, Christophe Fiter ${ }^{\mathrm{a}}$, Laurentiu Hetel ${ }^{\mathrm{a}}$, Nathan van de Wouw ${ }^{\mathrm{b}, \mathrm{c}}$, \\ Jean-Pierre Richard ${ }^{a}$ \\ ${ }^{\mathrm{a}}$ Univ. Lille, CNRS, Inria, Centrale Lille, UMR 9189 - CRIStAL, F-59000 Lille, France \\ ${ }^{\mathrm{b}}$ Eindhoven University of Technology, Eindhoven $5600 \mathrm{MB}$, The Netherlands \\ ${ }^{\mathrm{c}}$ Department of Civil, Environmental and Geo-Engineering, University of Minnesota, Minneapolis, MN 55455 USA
}

\begin{abstract}
In this paper, we provide novel conditions for stability analysis of aperiodically sampled nonlinear control systems subjected to time-varying delay. The proposed approach can also deal with cases in which delay is larger than the sampling interval. It is applicable to a general class of nonlinear systems and provides sufficient criteria for stability that aid in making trade-offs between control performance and the bounds on sampling interval and delay. As a stepping stone, a preliminary and generic result based on dissipativity, is introduced to analyse the exponential stability of a class of feedback-interconnected systems. The nonlinear sampled-data system is remodelled to consider the effects of sampling and delay in the dissipativity framework, as perturbations to the nominal closed-loop system. This leads to constructive stability conditions for a continuous time closed-loop system given by the feedback interconnection of the nominal closed-loop system and an operator(s) that captures the effects of sampling and delay. For Linear Time-Invariant (LTI) systems, we recover simple Linear Matrix Inequality (LMI) and frequency domain conditions previously proposed in the robust control framework.
\end{abstract}

Key words: Nonlinear sampled-data systems; Dissipativity; Time-varying delay; Stability analysis

\section{Introduction}

Currently, almost all sampled-data control systems are implemented numerically, and embedded in a networked environment where data is exchanged between sensors, controllers and actuators through digital communication channels [11, 35]. Examples include mobile sensor networks, smart grids, highway systems, etc., see [11].

However, in such control configurations, perturbing effects such as sampling jitter, data-packet dropouts, delays, etc., are often introduced in the network and this

\footnotetext{
^ This paper was not presented at any IFAC meeting. Corresponding author Jijju Thomas. Tel. +33-603763592.

Email addresses: j.thomas@tue.nl (Jijju Thomas), christophe.fiter@univ-lille.fr (Christophe Fiter), laurentiu.hetel@centralelille.fr (Laurentiu Hetel), nvd@tue.nl (Nathan van de Wouw),

jean-pierre.richard@centralelille.fr (Jean-Pierre Richard).
}

impacts the overall stability of the system $[1,35,11,9$, 12]. From the point of view of control theory, such phenomena are considered as sampled-data systems with aperiodic sampling and/or time-varying delay, or more generally, as Networked Control Systems (NCS) [35]. In this paper, we focus on the stability analysis problem for aperiodically sampled nonlinear systems subjected to time-varying delay.

Existing literature provides various methods that deal with the stability analysis of sampled-data systems, with or without delay. An overview of different approaches in the case of aperiodic sampled-data systems can be found in [12]. These approaches are broadly classified into four categories, i.e., the Time-delay approach, the Discretetime approach, the Hybrid systems approach, and the Input-output approach. The Time-delay approach, has been largely used in the context of Linear Time Invariant (LTI) systems [28]. One of the advantages of this approach is that it can easily handle situations in which 
delay is greater than sampling period [31]. However, it is usually difficult to make a differentiation between sampling induced delay and actuation induced delay. The approach has also been extended to nonlinear systems $[15,19]$. The Discrete-time approach, has been used for stability analysis of LTI systems $[6,8,31]$ and in some cases, nonlinear systems $[32,25]$. Since it is based on the exact system discretization, it leads to very accurate numerical tools for stability analysis. However, intersampling behaviour has been taken into account only in the case of LTI systems, see for example, [5]. Additionally, the application of such discretization-based approach is challenging for general nonlinear systems and for the large-delay case, see [18, 26]. The Hybrid system approach, was developed based on the fact that systems with sampling-and-hold in control and sensor signals can be modelled using impulsive systems [10]. In the LTI systems case, by using Impulsive Delay Differential Equations, situations when delay is greater than the sampling interval were also studied [16]. However, for nonlinear systems, the analysis has only been done for cases in which delay is less than the sampling interval $[2,27]$.

The Input-output approach, treats the error induced by sampling and/or delay as a perturbation to the continuous-time control system and captures its effects using an operator $[13,30]$. This approach is intuitively simple to develop and the stability analysis problem is related to the classical robust control framework [20,9]. A primary advantage of this approach is that it can easily include perturbations as well as nonlinearities. However, in the case of LTI systems, this approach has been used for stability analysis in the presence of sampling, and delay, only separately. The existing results only provide $\mathcal{L}_{2}$-stability criteria for LTI systems. Generally, it can be shown that this implies asymptotic stability of the LTI sampled-data system. However, in such cases, it is difficult to describe the system performance, even in terms of the transient decay-rate. In the case of nonlinear systems, this approach has been employed to analyse stability only in the case of aperiodic sampling in the absence of delay [23]. Providing constructive conditions for stability of nonlinear systems with aperiodic sampling and time-varying delay is largely an open problem.

In this paper, we provide a novel framework to analyse the stability of aperiodically sampled nonlinear systems subjected to time-varying delay, using an approach inspired from the notion of dissipativity [33]. The main contributions of this paper are as follows. We introduce a constructive approach that is applicable to a general class of aperiodically sampled nonlinear systems with time-varying delays, even in the scenario when delay is greater than the sampling interval. We provide two tractable exponential stability conditions by taking into account the specific discontinuities in delay, as well as inter-sampling and inter-actuation behaviour. The dissipativity-based approach proposed in this paper leads to conditions in terms of dissipativity type properties of the associated continuous-time system, for which many results for classes of nonlinear systems exist in literature. Additionally, the approach provides bounds on operator(s) characterizing sampling, hold and delay effects. The proposed results also aid in deciding the trade-off between system decay-rate, and the bounds on sampling interval and delay. As a stepping stone, we introduce a primary result that provides exponential stability conditions for a class of feedback interconnected systems, which bear relevance to a range of problems in the robust control framework. The first criterion caters to the so-called 'large delay case', which delineates the situation arising often in information transmission over shared networks, where the delay introduced to the data packet exceeds the sampling interval of the sensors. The second criterion, a less conservative one, deals with the 'small delay case' where delay is less than the sampling period. This scenario has been studied in numerous theoretical as well as practical settings (see [35, 34, 5]). For example, in [5], it was shown that in the case of a single sensor sampling periodically, when the sampled-data experienced delays less than sampling-interval, the system was rendered unstable. The problem becomes much more complex when the sensors and actuators involved have aperiodic sampling and actuation frequencies. In our analysis for the small-delay case, two separate operators are used to capture the effects of sampling and delay. In the case of LTI systems, we recover simple LMI and frequency domain conditions previously proposed in the robust control framework $[13,20]$.

The outline of this paper is as follows. In Section 2, we introduce the problem setting which comprises of a generic aperiodically sampled nonlinear system subjected to time-delay. In Section 3, a preliminary stability result in the exponential dissipativity framework is provided, for a class of feedback interconnected systems. Section 4 deals with the stability analysis of the nonlinear sampled-data system under the large-delay case. It begins with a model reformulation of the problem setting in terms of the feedback interconnection introduced in Section 3. Next, the remodelled system properties are exploited to formulate a required supply function that will be used to provide a stability criterion by employing the result introduced in Section 3. Section 5 introduces the stability analysis of the nonlinear sampled-data system in the small-delay case, and follows a similar outline as Section 4. In Section 6, examples are provided to corroborate the effectiveness of the proposed results in the nonlinear as well as linear case. Finally, conclusions and an insight into possible future work are given in Section 7. The proofs of the results introduced in this paper, if not given in the main body of the paper, are given in the appendices.

Notations: Throughout the paper, we denote $\mathbb{R}^{+}=$ $\{x \in \mathbb{R}: x \geq 0\}$. The Euclidean norm of a vector $x \in \mathbb{R}^{n}$ is denoted by $\|x\|$. For a time-varying vector $z(t) \in \mathbb{R}^{n}, \dot{z}(t)$ is the Dini derivative given by 
$\dot{z}(t) \triangleq \lim _{h \rightarrow 0^{+}} \sup \frac{z(t+h)-z(t)}{h}$. We denote $\mathcal{W}^{n}$ as the set of all piecewise continuous $n$-dimensional functions over $\mathbb{R}^{+}$. The notation $\mathbb{N}^{\star}$ is used to denote the set $\{\mathbb{N} \backslash\{0\}\}$. The set of all continuously differentiable functions is denoted by $\mathcal{C}^{1}$, and the set of all continuous functions are denoted by $\mathcal{C}^{0}$. The maximum and minimum eigen values of a matrix $M \in \mathbb{R}^{n \times n}$ are denoted by $\delta_{\max }$ and $\delta_{\text {min }}$, respectively. The Euclidean norm of a matrix $M$ is given by $\|M\|_{2}=\sqrt{\delta_{\max }\left(M^{T} M\right)}$.

\section{Problem Statement}

Consider the nonlinear system

$$
\dot{x}_{p}(t)=f\left(x_{p}(t)\right)+g\left(x_{p}(t)\right) u(t), \forall t \geq 0,
$$

with the nonlinear sampled-data control

$$
u(t)= \begin{cases}0, & \forall t \in\left[0, a_{0}\right), \\ \kappa\left(x_{p}\left(s_{k}\right)\right), & \forall t \in\left[a_{k}, a_{k+1}\right), k \in \mathbb{N},\end{cases}
$$

where $x_{p}(t) \in \mathbb{R}^{n_{p}}$ is the system state, and $u(t) \in \mathbb{R}^{m_{p}}$ is the control input based on the continuous time signal

$$
u_{c}(t)=\kappa\left(x_{p}(t)\right), \forall t \geq 0,
$$

subjected to sampling and delay. It is assumed that in the absence of sampling and delay, the origin of system (1) with $u(t)=u_{c}(t)$, is exponentially stable. The functions $f: \mathbb{R}^{n_{p}} \mapsto \mathbb{R}^{n_{p}}$ with $f(0)=0, g: \mathbb{R}^{n_{p}} \mapsto \mathbb{R}^{n_{p} \times m_{p}}$ are globally Lipschitz, and the function $\kappa: \mathbb{R}^{n_{p}} \mapsto \mathbb{R}^{m_{p}}$ belongs to $\mathcal{C}^{1}$. The time instants $s_{k}$ and $a_{k}$ specify the sampling instants (when sensors send the measured state value to the controller) and actuation instants (when the control input is updated at the actuator level) respectively. We consider a sampling sequence $\left\{s_{k}\right\}_{k \in \mathbb{N}}$ satisfying

$$
s_{k+1}=s_{k}+h_{k}, \forall k \in \mathbb{N}
$$

where the time-varying sampling interval $h_{k}$ satisfies $0<$ $\underline{h} \leq h_{k} \leq \bar{h}, \forall k \in \mathbb{N}$. Similarly, we consider the actuation sequence $\left\{a_{k}\right\}_{k \in \mathbb{N}}$ such that

$$
a_{k}=s_{k}+\tau_{k}, \forall k \in \mathbb{N},
$$

where $\tau_{k}$ is the time-varying delay between sampling and actuation instants and satisfies $0 \leq \tau \leq \tau_{k} \leq \bar{\tau}, \forall k \in \mathbb{N}$.

Hypothesis 1: The actuation instants satisfy

$$
a_{k}<a_{k+1}, \forall k \in \mathbb{N}
$$

This assumption allows the bound on delay, $\bar{\tau}$, to be greater than the bound on sampling interval, $\bar{h}$, but under the constraint that the actuation instants occur in an order corresponding to the sampling instants. Without loss of generality, we consider that the first actuation occurs at time $a_{0}=\bar{\tau}+\bar{h}$, while the first sampling instant is $s_{0}=a_{0}-\tau_{0}$. This assumption can also be ensured with a time-scale shift. Throughout the paper, $\mathcal{P}$ denotes the nonlinear closed-loop sampled-data system defined by (1), (2), (4)-(6). The objective of this paper is to analyse the exponential stability of the system $\mathcal{P}$.

\section{Preliminary Generic Stability Result}

In this paper, we will use the fact that system $\mathcal{P}$ can be remodelled as the feedback-interconnection given by

$$
\Sigma:\left\{\begin{array}{l}
\dot{x}(t)=\bar{f}_{0}(x(t)) \\
\left.y(t)=\bar{h}_{0}(x(t))\right\} \forall t \in\left[0, a_{0}\right), \\
\dot{x}(t)=\bar{f}(x(t))+\bar{g}(x(t)) \omega(t) \\
y(t)=\bar{h}(x(t))+\bar{l}(x(t)) \omega(t)
\end{array}\right\} \forall t \geq a_{0},
$$

with $x(t) \in \mathbb{R}^{n}, \omega(t) \in \mathbb{R}^{m}, y(t) \in \mathbb{R}^{p}, x(0)=x_{0}$, and the operator $\Delta: \mathcal{W}^{p} \mapsto \mathcal{W}^{m}$ such that

$$
\omega=\Delta y
$$

The function $\bar{f}_{0}$ in $(7)$ is considered to be globally Lipschitz, with a Lipschitz constant $k_{0}$ and $\bar{f}_{0}(0)=0$. Additionally, we consider that the functions $\bar{f}, \bar{g}, \bar{h}$ and $\bar{l}$ are sufficiently smooth. We assume that solutions exist for the feedback interconnection $\Sigma-\Delta$. We shall denote the feedback interconnection (7)-(8) by $\Sigma-\Delta$. Such interconnection models will be introduced in Sections 4 and 5 , wherein the functions introduced in (7) will also be detected. This will also establish the relation between the dimensions $n$ introduced in (7) and $n_{p}$ introduced in (1). Prior to presenting such models, we will formulate, a technical result concerning exponential stability of $\Sigma-\Delta$. This result will serve as a stepping stone for the stability analysis of systems of the form (1), (2), (4)-(6).

Theorem 1 Consider the feedback interconnection $\Sigma-$ $\Delta$ and the following assumptions:

Assumption 1: There exists a supply function $\mathcal{S}: \mathbb{R}^{+} \times$ $\overline{\mathbb{R}^{p} \times \mathbb{R}^{m} \mapsto \mathbb{R}}$ continuous in all parameters satisfying the integral constraint

$$
\int_{0}^{t} \mathcal{S}(\theta, \phi(\theta),(\Delta \phi)(\theta)) d \theta \leq 0, \forall t \geq 0, \phi \in \mathcal{W}^{p}
$$

Assumption 2: There exists a continuously differentiable storage function $V: \mathbb{R}^{n} \mapsto \mathbb{R}^{+}$and scalars $0<c_{1}<c_{2}$, and $q>0$ such that

$$
c_{1}\|x\|^{q} \leq V(x) \leq c_{2}\|x\|^{q} .
$$

Assumption 3: There exist scalars $\lambda \in \mathbb{R}$ and $\rho>0$ such that the inequalities

$$
-\mathcal{S}(t, y(t), \omega(t)) \leq \rho V(x(t)), \forall t \in\left[0, a_{0}\right),
$$




$$
\begin{aligned}
\dot{V}(x(t)) & \geq \lambda V(x(t)), t \in\left[0, a_{0}\right), \\
\dot{V}(x(t))+\alpha V(x(t)) & \leq e^{-\alpha\left(t-a_{0}\right)} \mathcal{S}(t, y(t), \omega(t)), \forall t \geq a_{0},
\end{aligned}
$$

are satisfied for some $\alpha>0$, along the solutions of the system $\Sigma-\Delta$.

Then $\Sigma-\Delta$ is exponentially stable with a decay-rate of at least $\alpha / q$, i.e., $\exists \delta>0: \forall t \geq 0,\|x(t)\| \leq \delta e^{\frac{-\alpha}{q} t}\|x(0)\|$.

Inequality (13) is motivated from the notion of exponential dissipativity introduced in [4], wherein exponentially weighted storage and supply functions were used to establish exponential stability conditions for nonlinear dynamical systems. The aforementioned theorem is a general result for stability analysis of feedback interconnected systems of the form $\Sigma-\Delta$. However, it also applies to the robustness analysis of systems subjected to various perturbations that can be modelled by an operator of the form (8).

Remark: If the assumptions in Theorem 1 only hold locally, the results can be extended easily in a manner similar to the one shown in [23], so that the conditions hold in a compact set containing the origin. Note that the result provided in [23] holds only for scenarios with aperiodic sampling alone. Theorem 1 generalizes the result in [23] by taking into account a general class of perturbation characterizing the effects of sampling and delay. The following sections explain how Theorem 1 allows for building robust stability critera for the nonlinear sampled-data system $\mathcal{P}$. In Section 4 , we consider the large delay case given by Hypothesis 1, i.e. (6). Similarly, in Section 5, we provide stability conditions for the small delay case, given by $\tau_{k}<h_{k}, \forall k \in \mathbb{N}$.

\section{Stability Analysis for the Large Delay Case}

In this section, we provide a constructive approach for applying Theorem 1 to analyse the stability of system $\mathcal{P}$ introduced in Section 2. The term 'large delay' signifies Hypothesis 1, which implies that the delay $\tau_{k}$ can indeed be greater than the sampling interval $h_{k}$, under the constraint that the actuation instants occur in order. Theorem 1 can be used in this scenario by reformulating the system $\mathcal{P}$ as an interconnection of the form $\Sigma-\Delta$ given by (7)-(8), so that the effects of sampling and delay are included as a perturbation. In order to do so, we define the perturbation induced by sampling and delay as

$$
e(t)=\left\{\begin{array}{l}
0, \forall t \in\left[0, a_{0}\right), \\
\kappa\left(x_{p}\left(s_{k}\right)\right)-\kappa\left(x_{p}(t)\right), \forall t \in\left[a_{k}, a_{k+1}\right), k \in \mathbb{N} .
\end{array}\right.
$$

For all $t \geq a_{0}, e(t)$ can be interpreted as the 'error' on the control action when compared to a continuous time controller as given in (3). We will introduce an operator $\Delta$ that helps in expressing the error $e(t)$ in an alternate manner. Additionally, we provide the functions introduced in (7), so that the dynamics of the interconnection $\Sigma-\Delta$ and the sampled-data system $\mathcal{P}$ are equivalent.

\subsection{System Model Reformulation}

In this section, we introduce a particular case of operator $\Delta$ in (8), with $m=p=m_{p}$, that captures the perturbation (14). Subsequently, the system $\mathcal{P}$ given by (1), $(2),(4)-(6)$ is reformulated in terms of a feedback interconnection of the form $\Sigma-\Delta$ in (7), (8).

Lemma 2 Consider the operator $\Delta: \mathcal{W}^{m_{p}} \mapsto \mathcal{W}^{m_{p}}$ defined for any signal $z \in \mathcal{W}^{m_{p}}$ as

$$
(\Delta z)(t)=\left\{\begin{array}{l}
0, \forall t \in\left[0, a_{0}\right) \\
-\int_{s_{k}}^{t} z(s) d s, \forall t \in\left[a_{k}, a_{k+1}\right), k \in \mathbb{N}
\end{array}\right.
$$

and the derivative of the continuous control in (3),

$$
\dot{u}_{c}(t)=\frac{d}{d t} \kappa\left(x_{p}(t)\right)
$$

Then, the sampling and delay induced error e defined in (14) can be expressed as $e=\Delta \dot{u}_{c}$.

We show next how the sampled-data system $\mathcal{P}$ can be remodelled in the format $\Sigma-\Delta$ given by (7), (8). This formulation in conjunction with Lemma 2 is used to prove the equivalence between the sampled-data system $\mathcal{P}$ and the interconnection $\Sigma-\Delta$.

Lemma 3 Consider the system $\Sigma$ in (7), with

$$
\begin{aligned}
& \bar{f}_{0}(x)=f(x), \bar{h}_{0}(x)=\frac{\partial \kappa(x)}{\partial x} \bar{f}_{0}(x), \\
& \bar{f}(x)=f(x)+g(x) \kappa(x) \\
& \bar{g}(x)=g(x), \bar{h}(x)=\frac{\partial \kappa(x)}{\partial x} \bar{f}(x), \bar{l}(x)=\frac{\partial \kappa(x)}{\partial x} \bar{g}(x),
\end{aligned}
$$

$n=n_{p}, m=p=m_{p}$, and the operator $\Delta$ in (8), defined by (15). Then, system $\mathcal{P}$ can be expressed as the feedback interconnection $\Sigma-\Delta$ in (7), (8), with $x=x_{p}$.

Remark: Modelling system (1), (2) in the form of (7), (8) implies adding an artificial output $y$, that will correspond to the derivative of the continuous-time control input, as given in (16).

Lemmas 2 and 3 will be used to provide constructive stability conditions for the system $\mathcal{P}$. In the following section, as a prerequisite for this development, the properties of $\Delta$ in (15) are exploited to provide a supply function $\mathcal{S}$ that satisfies the assumptions in Theorem 1 .

\subsection{Stability Analysis}

In this section, we characterize the properties of $\Delta$ by a supply function $\mathcal{S}$ satisfying assumption (9). 
Lemma 4 Consider $\Delta$ defined in (15), $\alpha \in \mathbb{R}^{+}$and $R \in$ $\mathbb{R}^{m_{p} \times m_{p}}$ with $R=R^{T}>0$. Then, for all $z \in \mathcal{W}^{m_{p}}$,

$$
\int_{0}^{t} \mathcal{S}(\theta, z(\theta),(\Delta z)(\theta)) d \theta \leq 0, \quad \forall t \geq 0
$$

where the function $\mathcal{S}: \mathbb{R}^{+} \times \mathbb{R}^{m_{p}} \times \mathbb{R}^{m_{p}} \mapsto \mathbb{R}$ is defined by

$$
\mathcal{S}:(\theta, v, w) \mapsto e^{\alpha\left(\theta-a_{0}\right)}\left(w^{T} R w-\gamma^{2} v^{T} R v\right)
$$

with $\gamma^{2}=(\bar{h}+\bar{\tau})^{2} e^{\alpha(\bar{h}+\bar{\tau})}$.

The result presented in Lemma 4 holds for any symmetric positive definite matrix $R$ characterizing the supply function. The following Theorems 5 and 6 , provide tools to tune the matrix $R$. The supply function given by (19), together with Lemmas 2 and 3, can now be used to provide stability conditions for the sampled-data system $\mathcal{P}$.

Theorem 5 Consider system $\mathcal{P}$ in (1), (2), (4)-(6), the interconnection $\Sigma-\Delta$ given by (7), (8), (15) and (17). If there exists a supply function $\mathcal{S}$ of the form (19) and a storage function $V: \mathbb{R}^{n} \mapsto \mathbb{R}^{+}$that satisfy assumptions (10), (11), (12) and (13), then system $\mathcal{P}$ is exponentially stable with a decay-rate $\alpha / q$.

Proof First, we exploit Lemma 3 to show the equivalence between $\mathcal{P}$ in (1), (2), (4)-(6) and $\Sigma-\Delta$ in (7), (8). Then, by Lemma 4, Assumption 1 in Theorem 1 is satisfied for the operator $\Delta$ defined by (15). Under the conditions of the theorem, Assumptions 2 and 3 of Theorem 1 are satisfied. Applying Theorem $1, \Sigma-\Delta$ is proved to be exponentially stable and therefore, so is system $\mathcal{P}$

Remark: The aforementioned theorem provides (only) sufficient stability conditions based on the existence of a storage function. In the following sections, we will present how this can be used in a constructive manner based on LMI and Sum of Squares (SOS) criteria. In Section 6 , we will illustrate with examples, how Theorem 5 can be used to provide stability conditions for nonlinear sampled-data systems of the form given by $\mathcal{P}$. In Section 6.1, for an exemplary nonlinear system, we will show how the matrix $R$ characterizing the supply function, can be tuned using standard MATLAB routines.

\subsection{Stability Criterion for Linear Systems}

Consider the linear sampled-data system $\mathcal{P}_{L}$ given by

$$
\dot{x}(t)=A x(t)+B u(t), \forall t \geq 0,
$$

with

$$
u(t)= \begin{cases}0, & \forall t \in\left[0, a_{0}\right), \\ K x\left(s_{k}\right), & \forall t \in\left[a_{k}, a_{k+1}\right), k \in \mathbb{N},\end{cases}
$$

where $x(t) \in \mathbb{R}^{n}, A \in \mathbb{R}^{n \times n}, B \in \mathbb{R}^{n \times m}$, and $K \in \mathbb{R}^{m \times n}$. Now, we provide a stability criterion for the linear sampled-data system $\mathcal{P}_{L}$ in the form of tractable LMI.

Theorem 6 Consider $\alpha \in \mathbb{R}^{+}$. The linear sampled-data system $\mathcal{P}_{L}$ is exponentially stable with a decay-rate $\alpha / 2$ if there exists $P=P^{T}>0$ and $R=R^{T}>0$ such that

$$
\left[\begin{array}{cc}
\bar{A}^{T} P+P \bar{A}+\alpha P & P B \\
B^{T} P & 0
\end{array}\right]+\left[\begin{array}{cc}
K \bar{A} & K B \\
0 & I
\end{array}\right]^{T}\left[\begin{array}{cc}
\gamma^{2} R & 0 \\
0 & -R
\end{array}\right]\left[\begin{array}{cc}
K \bar{A} & K B \\
0 & I
\end{array}\right]<0
$$

with $\bar{A}=A+B K$, and $\gamma^{2}=(\bar{h}+\bar{\tau})^{2} e^{\alpha(\bar{h}+\bar{\tau})}$.

Remark: Applying the Kalman-Yakubovich-Popov Lemma, we can infer that the LMI given by (22) is equivalent to the frequency-domain criterion $\|\tilde{G}\|_{\infty}<1 / \gamma$, where $\tilde{G}$ is the operator defined by the transfer function $\tilde{G}(s)=K \bar{A}\left(s I-\bar{A}-\frac{\alpha}{2} I\right)^{-1} B+K B$. This result is in fact a generalization of the results provided in [13] and [20]. We have extended the results in $[13,20]$ by providing stability conditions for non-linear sampled-data systems while guaranteeing an exponential decay-rate. If $\alpha=0$, and $\bar{h}=0$, we recover the result in [13]. Similarly, if $\alpha=0$, and $\bar{\tau}=0$, we recover the result provided in [20]. In Section 6.2, we will demonstrate how matrices $P$ and $R$ can be tuned numerically using standard LMI solvers.

\section{Stability Analysis for the Small Delay Case}

The large-delay case studied in Section 4 is more generic to processes communicating via a shared network, where traffic flow can increase considerably. However, in some cases, it has been shown that it is desirable to have delay less than sampling interval since sampled data arriving in a non-chronological order at the actuator can be hazardous from a control point of view [1]. Consequentially, this would make the implementations of algorithms and analysis much more complex. In this section, we will demonstrate how considering sampling and delay separately in the small-delay case, gives a less conservative stability criterion. The following assumption is considered throughout the section.

Hypothesis 2: The actuation based on the sampled state $x\left(s_{k}\right)$ is implemented before the next sampling instant $s_{k+1}$, i.e.,

$$
\tau_{k}<h_{k}, \forall k \in \mathbb{N}
$$

Next, we re-formulate the sampled-data model for system $\mathcal{P}$ in order to include the effects of sampling and delay using two separate errors, denoted by $e_{s}(t)$ and $e_{d}(t)$, respectively. Consider the continuous-time control $u_{c}(t)=\kappa\left(x_{p}(t)\right)$. The sampled version of this signal is $u_{s}(t)=\kappa\left(x_{p}\left(s_{k}\right)\right), \forall t \in\left[s_{k}, s_{k+1}\right), k \in \mathbb{N}$. The samplinginduced error $e_{s}(t)$ is $e_{s}(t)=u_{s}(t)-u_{c}(t)$. Without loss 
of generality, we consider that $e_{s}(t)=0, \forall t<s_{0}$. Then,

$$
e_{s}(t)=\left\{\begin{array}{l}
0, \forall t \in\left[0, s_{0}\right), \\
\kappa\left(x_{p}\left(s_{k}\right)\right)-\kappa\left(x_{p}(t)\right), \forall t \in\left[s_{k}, s_{k+1}\right), k \in \mathbb{N} .
\end{array}\right.
$$

The delayed version of $u_{s}(t)$ is the control signal $u(t)$ applied at the level of the actuator. We introduce another error $e_{d}(t)$, which can be given by $u(t)-u_{s}(t)$. Note that we can define the error $e_{d}(t)=0, \forall t<a_{0}$, since it bears no relevance. Formally, $e_{d}(t)$ is given by

$$
e_{d}(t)=\left\{\begin{array}{l}
0, \forall t \in\left[0, a_{0}\right), \\
0, \forall t \in\left[a_{k-1}, s_{k}\right), k \in \mathbb{N}^{\star}, \\
\kappa\left(x_{p}\left(s_{k-1}\right)\right)-\kappa\left(x_{p}\left(s_{k}\right)\right), \forall t \in\left[s_{k}, a_{k}\right), k \in \mathbb{N}^{\star}
\end{array}\right.
$$

Using this formulation for $e_{s}(t)$ and $e_{d}(t)$, given by (24) and (25), respectively, we proceed to reformulate the sampled-data system $\mathcal{P}$ in the form of $\Sigma-\Delta$.

\subsection{System Model Reformulation}

In this section, we introduce two different operators $\Delta_{s}$ and $\Delta_{d}$, which capture the errors induced by sampling and delay given in (24) and (25), respectively. In an approach similar to the one used in Section 4.1, system $\mathcal{P}$ under Hypothesis 2, i.e. (23), can be represented as a feedback interconnection of the form $\Sigma-\Delta$.

Lemma 7 Consider the operator $\Delta: \mathcal{W}^{2 m_{p}} \mapsto \mathcal{W}^{2 m_{p}}$

$$
\Delta: \phi=\left(\begin{array}{c}
v \\
w
\end{array}\right) \rightarrow(\Delta \phi)=\left(\begin{array}{c}
\Delta_{s} v \\
\Delta_{d} w
\end{array}\right), \forall v \in \mathcal{W}^{m_{p}}, w \in \mathcal{W}^{m_{p}}
$$

under Hypothesis 2, i.e. (23), where

$$
\left(\Delta_{s} v\right)(t)=\left\{\begin{array}{l}
0, \forall t \in\left[0, s_{0}\right), \\
-\int_{s_{k}}^{t} v(\theta) d \theta, \forall t \in\left[s_{k}, s_{k+1}\right), k \in \mathbb{N},
\end{array}\right.
$$

and

$$
\left(\Delta_{d} w\right)(t)=\left\{\begin{array}{l}
0, \forall t \in\left[0, a_{0}\right) \\
0, \forall t \in\left[a_{k-1}, s_{k}\right), k \in \mathbb{N}^{\star} \\
-\int_{s_{k-1}}^{s_{k}} w(\theta) d \theta, \forall t \in\left[s_{k}, a_{k}\right), k \in \mathbb{N}^{\star}
\end{array}\right.
$$

Then, the sampling and delay induced errors defined in (24) and (25), respectively, can be expressed as

$$
\left(\begin{array}{l}
e_{s} \\
e_{d}
\end{array}\right)=\left(\begin{array}{c}
\Delta_{s} \dot{u}_{c} \\
\Delta_{d} \dot{u}_{c}
\end{array}\right)
$$

with $\dot{u}_{c}$ given by (16).

Analogous to the approach used in Section 4, we now proceed to reformulate the sampled-data system $\mathcal{P}$ under
Hypothesis 2 , i.e. (23), in the format $\Sigma-\Delta$ given by (7), (8). In the following lemma, by using such a model reformulation along with Lemma 7, we provide the equivalence between the sampled-data system $\mathcal{P}$ under Hypothesis 2 , and the feedback interconnection $\Sigma-\Delta$.

Lemma 8 Consider the system $\Sigma$ in (7), with

$$
\begin{aligned}
& \bar{f}_{0}(x)=f(x), \bar{h}_{0}(x)=\left[\begin{array}{l}
\frac{\partial \kappa(x)}{\partial x} \bar{f}_{0}(x) \\
\frac{\partial \kappa(x)}{\partial x} \bar{f}_{0}(x)
\end{array}\right], \\
& \bar{f}(x)=f(x)+g(x) \kappa(x), \bar{g}(x)=[g(x) g(x)], \\
& \bar{h}(x)=\left[\begin{array}{l}
\frac{\partial \kappa(x)}{\partial x} \bar{f}(x) \\
\frac{\partial \kappa(x)}{\partial x} \bar{f}(x)
\end{array}\right], \bar{l}(x)=\left[\begin{array}{l}
\frac{\partial \kappa(x)}{\partial x} \bar{g}(x) \\
\frac{\partial \kappa(x)}{\partial x} \bar{g}(x)
\end{array}\right],
\end{aligned}
$$

$n=n_{p}, m=p=2 m_{p}$, and the operator $\Delta$ in (8), defined by (26), (27) and (28) under Hypothesis 2, i.e. (23). Then, the sampled-data system $\mathcal{P}$ can be expressed as the feedback interconnection $\Sigma-\Delta$, with $x=x_{p}$.

Lemmas 7 and 8 are used to provide constructive stability criterion for sampled-data system $\mathcal{P}$ under Hypothesis 2 . To this end, the supply function $\mathcal{S}$ given in Theorem 1 needs to be formulated. We proceed in this direction by studying the properties of operators $\Delta_{s}$ and $\Delta_{d}$.

\subsection{Stability Analysis}

In this section, we characterize the properties of $\Delta_{s}$ and $\Delta_{d}$, by functions $\mathcal{S}_{s}$ and $\mathcal{S}_{d}$, respectively. Consequently, we formulate the supply function $\mathcal{S}=\mathcal{S}_{s}+\mathcal{S}_{d}$.

Lemma 9 Consider the operator $\Delta_{s}$ defined in (27), $\beta \in$ $\mathbb{R}^{+}$and $R_{s} \in \mathbb{R}^{m_{p} \times m_{p}}$ with $R_{s}=R_{s}^{T}>0$. Then,

$$
\int_{0}^{t} \mathcal{S}_{s}\left(\theta, v(\theta),\left(\Delta_{s} v\right)(\theta)\right) d \theta \leq 0, \quad \forall t \geq 0, v \in \mathcal{W}^{m_{p}}
$$

where the function $\mathcal{S}_{s}: \mathbb{R}^{+} \times \mathbb{R}^{m_{p}} \times \mathbb{R}^{m_{p}} \mapsto \mathbb{R}$ is defined as

$$
\mathcal{S}_{s}:(\theta, v, \mu) \rightarrow e^{\beta\left(\theta-a_{0}\right)}\left[\begin{array}{l}
v \\
\mu
\end{array}\right]^{T}\left[\begin{array}{cc}
-\gamma_{s}^{2} R_{s} & \gamma_{s}^{2} \frac{\beta}{2} R_{s} \\
\gamma_{s}^{2} \frac{\beta}{2} R_{s} & \left(1-\gamma_{s}^{2} \frac{\beta^{2}}{4}\right) R_{s}
\end{array}\right]\left[\begin{array}{l}
v \\
\mu
\end{array}\right],
$$

with $\gamma_{s}=\frac{2 \bar{h}}{\pi}$.

Lemma 10 Consider $\Delta_{d}$ defined in (28) under Assumption 2, $\beta \in \mathbb{R}^{+}$and $R_{d} \in \mathbb{R}^{m_{p} \times m_{p}}$ with $R_{d}=R_{d}^{T}>0$. Then, for all $w \in \mathcal{W}^{m_{p}}$,

$$
\int_{0}^{t} \mathcal{S}_{d}\left(\theta, w(\theta),\left(\Delta_{d} w\right)(\theta)\right) d \theta \leq 0, \quad \forall t \geq 0
$$

where the function $\mathcal{S}_{d}: \mathbb{R}^{+} \times \mathbb{R}^{m_{p}} \times \mathbb{R}^{m_{p}} \mapsto \mathbb{R}$ is defined as

$$
\mathcal{S}_{d}:(\theta, w, \varepsilon) \rightarrow e^{\beta\left(\theta-a_{0}\right)}\left[\begin{array}{l}
w \\
\varepsilon
\end{array}\right]^{T}\left[\begin{array}{cc}
-\gamma_{d} R_{d} & 0 \\
0 & R_{d}
\end{array}\right]\left[\begin{array}{l}
w \\
\varepsilon
\end{array}\right],
$$


with $\gamma_{d}=\bar{h} \bar{\tau} e^{\beta(\bar{h}+\bar{\tau})}$

The functions $\mathcal{S}_{s}$ and $\mathcal{S}_{d}$ given in Lemmas 9 and 10, provide the sampling and delay component, respectively, of the supply function $\mathcal{S}=\mathcal{S}_{s}+\mathcal{S}_{d}$. As follows, we use the supply function $\mathcal{S}=\mathcal{S}_{s}+\mathcal{S}_{d}$ to provide a general, more accurate stability criterion for the sampled-data system $\mathcal{P}$ under Hypothesis 2, i.e., when delay is less than sampling interval.

Theorem 11 Consider system $\mathcal{P}$, the interconnection $\Sigma-\Delta$ given by (7), (8), (26), (27), (28) and (30). If there exist functions $\mathcal{S}=\mathcal{S}_{s}+\mathcal{S}_{d}$ defined using (32) and (34), and $V: \mathbb{R}^{n_{p}} \mapsto \mathbb{R}^{+}$that satisfy assumptions (10), (11), (12) and (13), then system $\mathcal{P}$ is exponentially stable with a decay-rate $\alpha / q$.

Proof We exploit Lemma 8 to establish the equivalence between system $\mathcal{P}$ under Hypothesis 2 and $\Sigma-\Delta$ in (7), (8). Then, by Lemmas 9 and 10, Assumption 1 in Theorem 1 is satisfied for the operator $\Delta$ defined by (26), (27) and (28). Under the conditions of the theorem, Assumptions 2 and 3 of Theorem 1 are satisfied. Applying Theorem $1, \Sigma-\Delta$ is proved to be exponentially stable and by equivalence, so is system $\mathcal{P}$.

The result presented in Theorem 6 holds for any positive symmetric definite matrices $R_{s}$ and $R_{d}$ characterizing the supply function. In Section 6.1, we will illustrate how Theorem 11 provides less conservative results for the sampled-data system $\mathcal{P}$ under Hypothesis 2, i.e., for the small delay case. The usage of numerical tools to tune matrices $R_{s}$ and $R_{d}$, will also be shown.

\subsection{Stability Criterion for Linear Systems}

In this section, we recall the linear sampled-data system $\mathcal{P}_{L}$ described in Section 4.3 by (20). Based on the Lemmas 9 and 10, we provide the following stability criterion for system $\mathcal{P}_{L}$ under Hypothesis 2 .

Theorem 12 Consider a scalar $\alpha \in \mathbb{R}^{+}$and Hypothesis 2. The linear sampled-data system $\mathcal{P}_{L}$ is exponentially stable with a decay-rate $\alpha / 2$ if there exist $P=P^{T}>0$, $R_{s}=R_{s}^{T}>0$, and $R_{d}=R_{d}^{T}>0$ such that

$$
\begin{gathered}
{\left[\begin{array}{cc}
\bar{A}^{T} P+P \bar{A}+\alpha P & P \bar{B} \\
\bar{B}^{T} P & 0
\end{array}\right]+\left[\begin{array}{cc}
K \bar{A} & K \bar{B} \\
0 & I
\end{array}\right]^{T} \Phi\left[\begin{array}{cc}
K \bar{A} & K \bar{B} \\
0 & I
\end{array}\right]<0,} \\
\text { with } \bar{A}=A+B K, \bar{B}=\left[\begin{array}{ll}
B & B
\end{array}\right] \text {, and } \\
\Phi=\left[\begin{array}{ccc}
\gamma_{s}^{2} R_{s}+\gamma_{d} R_{d} & -\gamma_{s}^{2} \frac{\alpha}{2} R_{s} & 0 \\
-\gamma_{s}^{2} \frac{\alpha}{2} R_{s} & \left(\gamma_{s}^{2} \frac{\alpha^{2}}{4}-1\right) R_{s} & 0 \\
0 & 0 & -R_{d}
\end{array}\right],
\end{gathered}
$$

where $\gamma_{s}=\frac{2 \bar{h}}{\pi}$ and $\gamma_{d}=\bar{h} \bar{\tau} e^{\alpha(\bar{h}+\bar{\tau})}$.

Remark: When $\alpha=0, \bar{\tau}=0$ (implying no delay component in $\mathcal{S}$ ), the LMI (35) translates to a form similar to LMI (22). Consequentially, by virtue of the KalmanYakubovich-Popov lemma, we can recover the frequency domain condition introduced in [20], i.e., $\|\tilde{G}\|_{\infty}<\frac{\pi}{2 \bar{h}}$, where $\tilde{G}$ is the operator defined by the transfer function $\tilde{G}(s)=K \bar{A}\left(s I-\bar{A}-\frac{\alpha}{2} I\right)^{-1} \bar{B}+K \bar{B}$.

In Section 6.2 , we will illustrate with examples, how the LMI (35) provides less conservative results for LTI systems under Hypothesis 2, i.e., for the small delay case.

\section{$6 \quad$ Illustrative Examples}

In this section, we illustrate the effectiveness of our proposed results via examples. The provided examples highlight the difference between the single-error approach and the separate-error approach in terms of conservativeness and trade-offs between control performance and the bounds on sampling interval and delay. The result presented in this paper provides a foundation for deciding the trade-off between maximum delay $\bar{\tau}$, maximum sampling period $\bar{h}$, and decay-rate $\alpha$. By fixing one of the parameters, the trade-off between the remaining parameters can be obtained. For example, by fixing $\bar{\tau}$, and gridding over $\bar{h}$ and $\alpha$, a trade-off between the decayrate and the maximum allowable sampling interval can be obtained. In a similar manner, fixing $\bar{h}$ will give the trade-off between $\alpha$ and $\bar{\tau}$, and so on.

\subsection{Nonlinear System Example}

We consider the following example [14, 21, 23],

$$
\dot{x}(t)=d x(t)^{2}-x(t)^{3}+u(t)
$$

with a bounded time-varying parameter $|d(t)| \leq 1$, and a stabilizing control $u(t)=\kappa(x(t))=-2 x(t)$ subjected to both sampling and delay. Since the function $f(x)=$ $x^{2}-x^{3}$ is locally Lipschitz, our results will only hold locally on any compact set containing the origin.

\subsubsection{Large-delay Case}

Using the definition in (17), we reformulate the system model in the form $\Sigma-\Delta$, where $\Sigma$ is given by

$$
\begin{aligned}
& \left.\begin{array}{l}
\dot{x}(t)=d x^{2}(t)-x^{3}(t) \\
y(t)=-2\left(d x^{2}(t)-x^{3}(t)\right)
\end{array}\right\} \forall t \in\left[0, a_{0}\right), \\
& \left.\begin{array}{l}
\dot{x}(t)=d x^{2}(t)-x^{3}(t)-2 x(t)+w(t) \\
y(t)=-2\left(d x^{2}(t)-x^{3}(t)-2 x(t)+w(t)\right)
\end{array}\right\} \forall t \geq a_{0} .
\end{aligned}
$$

We use a storage function of the form $V(x)=a x^{2}+b x^{4}$ as given in [23]. Using (19), we obtain the supply function 


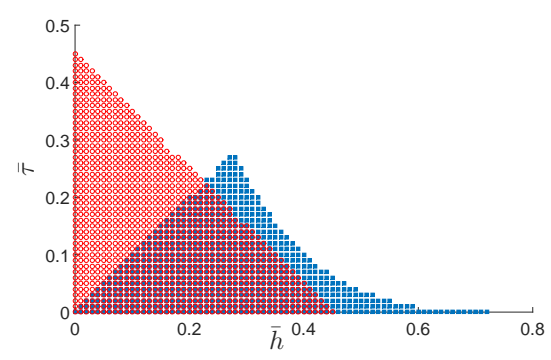

Fig. 1. Feasible values of $\bar{h}$ and $\bar{\tau}$ for the nonlinear system (37) with $\alpha=0.1$, in the large-delay case (in red), and in the small-delay case (in blue).

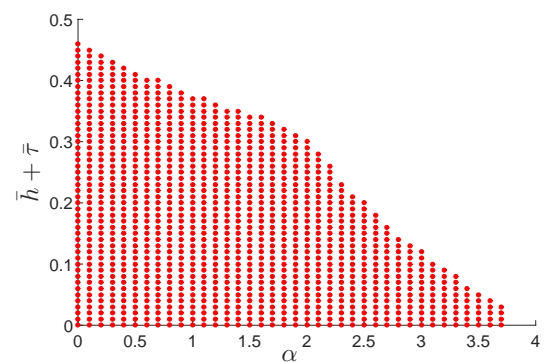

Fig. 2. Trade-off between desired decay-rate $\alpha$ and $\bar{h}+\bar{\tau}$ for the nonlinear system (37), in the large-delay case.

$\mathcal{S}(\theta, y, w)=e^{\alpha\left(\theta-a_{0}\right)}\left[R w^{2}(\theta)-\gamma^{2} R y^{2}(\theta)\right]$, with $\gamma^{2}=$ $(\bar{h}+\bar{\tau})^{2} e^{\alpha(\bar{h}+\bar{\tau})}$. For this case, from condition (13), we can infer that the values of $(\bar{h}+\bar{\tau})$ satisfying the inequality

$$
\begin{array}{r}
\left(2 a x+4 b x^{3}\right)\left(d x^{2}-x^{3}-2 x+w\right)+\alpha\left(a x^{2}+b x^{4}\right)-R w^{2} \\
+4(\bar{h}+\bar{\tau})^{2} e^{\alpha(\bar{h}+\bar{\tau})} R\left(d x^{2}-x^{3}-2 x+w\right)^{2} \leq 0,
\end{array}
$$

will guarantee exponential stability. If (39) can be expressed as a Sum of Squares (SOS) for all the values of $\left(d, d^{2}\right) \in\{(1,0),(1,1),(-1,0),(-1,1)\}$, then it will be SOS for any time-varying $|d(t)| \leq 1$. Using SOSTOOLS [24], Figure 1 provides the feasible values of $\bar{h}$ and $\bar{\tau}$ (in red) for $\alpha=0.1$, and all values of $\left(d, d^{2}\right)$. It can be seen from Figure 1 that, for $\alpha=0.1, \bar{h}$ and $\bar{\tau}$ satisfy a maximum bound $\bar{h}+\bar{\tau} \leq 0.45$, with $a=0.7079, b=0.1890$ and $R=0.4268$. The parameters $a, b$ and $R$ are optimized using SOSTOOLS. Additionally, the trade-off between the desired decay-rate $\alpha / 2$ and $\bar{h}+\bar{\tau}$ is shown in Figure 2 .

\subsubsection{Small-delay Case}

Now, we shall provide bounds on $\bar{h}$ and $\bar{\tau}$ in the smalldelay case, i.e., $\tau_{k}<h_{k}$. In this case, the system model is reformulated in the form $\Sigma$, given by

$$
\left.\begin{array}{l}
\dot{x}(t)=d x^{2}(t)-x^{3}(t) \\
y(t)=\left[\begin{array}{ll}
y_{1}(t) & y_{2}(t)
\end{array}\right]^{T}
\end{array}\right\} \forall t \in\left[0, a_{0}\right),
$$

with $y_{1}(t)=y_{2}(t)=-2\left(d x^{2}(t)-x^{3}(t)\right)$ and

$$
\begin{aligned}
& \dot{x}(t)=d x^{2}(t)-x^{3}(t)-2 x(t)+w_{s}(t)+w_{d}(t), \\
& y(t)=\left[y_{1}(t) y_{2}(t)\right]^{T}, \forall t \geq a_{0},
\end{aligned}
$$

where $y_{1}(t)=y_{2}(t)=-2\left(d x^{2}(t)-x^{3}(t)-2 x(t)+w_{s}(t)+\right.$ $\left.w_{d}(t)\right)$. Using (32) and (34), we get the supply function

$$
\begin{aligned}
\mathcal{S}(\theta, y, w)= & \mathcal{S}_{s}\left(\theta, y_{1}, w_{s}\right)+\mathcal{S}_{d}\left(\theta, y_{2}, w_{d}\right) \\
= & e^{\alpha\left(\theta-a_{0}\right)}\left[-\gamma_{s}^{2} R_{s} y_{1}^{2}(\theta)-\gamma_{d} R_{d} y_{2}^{2}(\theta)\right. \\
& +\gamma_{s}^{2} \alpha R_{s} y_{1}(\theta) w_{s}(\theta)+\left(1-\gamma_{s}^{2} \frac{\alpha^{2}}{4}\right) R_{s} w_{s}^{2} \\
& \left.+R_{d} w_{d}^{2}\right],
\end{aligned}
$$

where $\gamma_{s}=\frac{2 \bar{h}}{\pi}$ and $\gamma_{d}=\bar{h} \bar{\tau} e^{\beta(\bar{h}+\bar{\tau})}$. Therefore, by using the supply function (42) in condition (13), we must deduce the values of $\bar{h}$ and $\bar{\tau}$ satisfying the inequality

$$
\begin{gathered}
\left(2 a x+4 b x^{3}\right)\left(d x^{2}-x^{3}-2 x+w_{s}+w_{d}\right)+\alpha\left(a x^{2}+b x^{4}\right) \\
+4\left(\gamma_{s}^{2} R_{s}+\gamma_{d} R_{d}\right)\left(d x^{2}-x^{3}-2 x+w_{s}+w_{d}\right)^{2} \\
+2 \gamma_{s}^{2} \alpha R_{s}\left(d x^{2}-x^{3}-2 x+w_{s}+w_{d}\right) w_{s} \\
-\left(1-\gamma_{s}^{2} \frac{\alpha^{2}}{4}\right) R_{s} w_{s}^{2}-R_{d} w_{d}^{2} \leq 0
\end{gathered}
$$

in order to guarantee exponential stability of the system (37), with $\alpha>0, \tau_{k}<h_{k}$. For the sake of comparison with the feasibility region obtained in the large-delay case, we choose $\alpha=0.1$. In a similar manner as shown in the large-delay case, we use SOSTOOLS to obtain the feasible values of $\bar{h}$ and $\bar{\tau}$ satisfying inequality (43), for all values of $\left(d, d^{2}\right)$, while optimizing the values of $a, b$, $R_{s}$ and $R_{d}$. The feasibility plot in the small-delay case is given in Figure 1 (in blue). In Figure 1, it can be seen that the red feasibility plot (indicating feasibility for the large-delay case) and the blue feasibility plot (indicating the small-delay case) overlap. This overlapping region represents the feasible values of $\bar{h}$ and $\bar{\tau}$ obtained when the criterion (39) provided for the large-delay case, is applied to the small-delay case. In such scenarios, Theorem 11 and Theorem 12 always provide better results in comparison to the results given by Theorem 5 and Theorem 6 , respectively. In Figure 1 , when $\bar{\tau} \rightarrow 0$, we can see that bounds on $\bar{h}$ upto 0.72 are feasible while using the tools presented in the small-delay analysis. The tool presented in the large-delay case, on the other hand, accommodates $\bar{h}$ upto 0.45 , thereby implying an improvement of about $60 \%$ while using the result provided in the small-delay case. Additionally, when $\bar{h}=0.27$, the feasible values of $\bar{\tau}$ in the large and small-delay cases, are approximately upto 0.17 and 0.27 , respectively, showing an improvement of about $59 \%$. Using these numerical arguments, it can be concluded that for the small-delay case, capturing the effects of sampling and delay using two separate errors gives less conservative results. However, the amount of improvement in the small-delay case 
over the large-delay case depends on the parameter $\alpha$. We illustrate this in the following section for a linear system example.

Remark: The less-conservative nature of the results proposed in the small-delay case can also be justified from a theoretical perspective. In the large-delay case, the supply function was formulated using Jensen's inequality, which introduces conservativeness [3]. On the other hand, in the small-delay case, Wirtinger's inequality has been used. For this case, the improvement over Jensen's inequality is well known in the literature [29].

For the same example in the absence of delay, in [22] and [14], upper-bounds of 0.368 and 0.143 , respectively, were obtained for the sampling intervals without any performance guarantee. This is comparable to the small-delay case we have considered, with $\bar{\tau}=0$. Additionally, in [23], an upper-bound of 0.72 was proposed for the system (37) without delay, with $\alpha=0.1$. From the results proposed for the small-delay case, by setting $\bar{\tau}=0$, indicating sampling without any delay, we can see in Figure 1 that we obtain the same upper-bound of 0.72 on the sampling intervals, as proposed in [23], with $a=2.9153 \times 10^{-6}$, $b=7.29 \times 10^{-7}, R_{s}=1.6964 \times 10^{-6}$ and $R_{d}=1.2465$. However, our results have an added advantage that we provide tractable stability conditions for the nonlinear sampled-data system in the presence of time-varying delay.

\subsection{Linear System Example}

Consider the system (20) characterized by the parameters [36]

$$
A=\left[\begin{array}{ll}
1 & 3 \\
2 & 1
\end{array}\right], B=\left[\begin{array}{c}
1 \\
0.6
\end{array}\right], K=-\left[\begin{array}{ll}
1 & 6
\end{array}\right]
$$

By virtue of Theorem 6 , we can compute the maximum allowable values of $\bar{h}+\bar{\tau}$ with respect to $\alpha$ from the LMI (22). The LMI (22) is solved using YALMIP, by optimizing parameters $P$ and $R$, for different values of $\alpha$ and $\bar{h}+\bar{\tau}$. The feasibility region thus obtained will aid in deciding the trade-off between a desired decay rate while taking into account the maximum bounds on sampling interval and delay. Considering $\alpha \in\{0.01,1,2\}$, we obtain the bounds on $\bar{h}$ and $\bar{\tau}$ as shown in Figure 3 (in red solid, dashed and dotted lines). For the LTI system (44), if $\alpha=0$ and $\bar{h}=0$, we recover the bound on $\bar{\tau}$ as given in [13]. For the chosen values of $\alpha \in\{0.01,1,2\}$, we also compute the bounds on $\bar{h}$ and $\bar{\tau}$ in the small-delay case (as shown in Figure 3 in blue solid, dashed and dotted lines). Following a similar explanation as given in Section 6.1.2, we can conclude that for the small-delay case, differentiating the effects of sampling and delay using two separate errors, the LMI in (35) introduced in Theorem 12 provides less conservative results in comparison to the criterion provided in (22) (applied to the smalldelay case). Figure 3 also gives the dependence of the

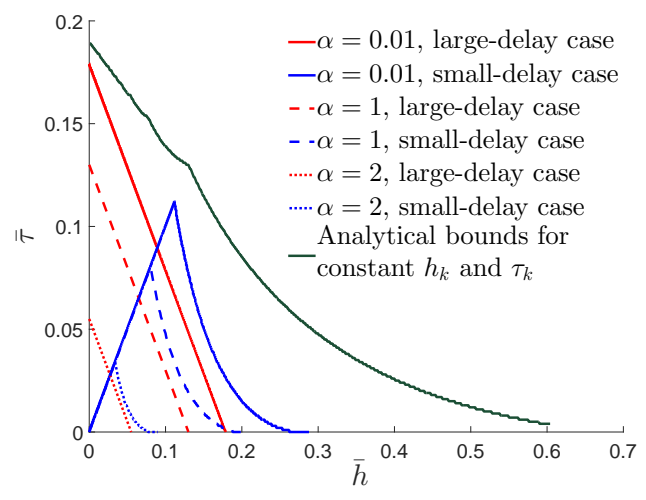

Fig. 3. Bounds on $\bar{h}$ and $\bar{\tau}$ for the LTI system (44) in the large-delay case (in red), and in the small-delay case (in blue), for $\alpha=0.01$ (solid line), $\alpha=1$ (dashed line) and $\alpha=2$ (dotted line). The analytical stability bounds on constant $h_{k}$ and $\tau_{k}$ are given by the green line [35].

amount of improvement in the small-delay case over the large-delay case, on the parameter $\alpha$. If $\alpha=0$ and $\bar{\tau}=0$, we recover the bound on $\bar{h}$ as proposed in [20]. Therefore, we can conclude that by applying our generic nonlinear tools to the linear case, we provide bounds on $\bar{h}$ and $\bar{\tau}$ that are not more conservative in comparison to the bounds provided in $[13,20]$. Also, it has to be noted that despite the fact that the condition in (22) is more conservative when applied to the small-delay case, the result is still important since it is applicable to the more generic large-delay case.

\section{Conclusion}

In this paper, novel approaches for stability analysis of aperiodically sampled nonlinear systems with timevarying delay are provided. The framework introduced in this paper holds for a general class of nonlinear systems and provides tools that help in deciding required tradeoffs between the system decay-rate and the bounds on sampling interval and delay. As a preliminary result, an approach inspired from the notion of exponential dissipativity is used to provide stability conditions for a class of feedback interconnected systems, while guaranteeing a desired decay-rate. The nonlinear sampled-data system is remodelled as a feedback interconnection of the nominal closed-loop system and an operator that captures the effects of sampling and delay, thereby leading to constructive stability conditions. The proposed approach leads to conditions on dissipativity properties of the system, for which many results exist in literature. When applying the results to LTI case, we see that they generalize existing frequency domain and LMI conditions in the robust stability framework. For the case when delay is less than sampling interval, a less conservative stability criterion is obtained by considering two separate operators to capture the effects of sampling and delay. The effectiveness of the proposed theoretical results have been corroborated via simulation results for 
an exemplary nonlinear system. We foresee numerous extensions. For example, a more realistic scenario would involve multiple sensors and actuators, each with unique bounds on sampling interval and delay $[30,7]$.

\section{Acknowledgements}

This work was supported by project UCoCoS, funded by the European Union's EU Framework Programme for Research and Innovation, Horizon H2020, Grant Agreement No: 675080

\section{References}

[1] K.J. Åström and B. Wittenmark. ComputerControlled Systems: Theory and Design. Prentice Hall, 1996.

[2] D.P. Borgers, R. Geiselhart, and W.P.M.H. Heemels. Tradeoffs between quality-of-control and quality-of-service in large-scale nonlinear networked control systems. Nonlinear Analysis: Hybrid Systems, 23:142 - 165, 2017.

[3] C. Briat. Convergence and equivalence results for the Jensen's inequality - Application to time-delay and sampled-data systems. IEEE Transactions on Automatic Control, 56(7):1660-1665, July 2011.

[4] V. Chellaboina and W. M. Haddad. Exponentially dissipative nonlinear dynamical systems: a nonlinear extension of strict positive realness. In Proceedings of the 2000 American Control Conference. ACC (IEEE Cat. No.00CH36334), volume 5, pages 3123-3127 vol.5, June 2000.

[5] M. Cloosterman, N. van de Wouw, M. Heemels, and H. Nijmeijer. Robust stability of networked control systems with time-varying network-induced delays. In Proceedings of the 45th IEEE Conference on Decision and Control, pages 4980-4985, Dec 2006.

[6] M.B.G. Cloosterman, L. Hetel, N. van de Wouw, W.P.M.H. Heemels, J. Daafouz, and H. Nijmeijer. Controller synthesis for networked control systems. Automatica, 46(10):1584 - 1594, 2010.

[7] C. Fiter, T. E. Korabi, L. Etienne, and L. Hetel. Stability of LTI systems with distributed sensors and aperiodic sampling. Control Subject to Computational and Communication Constraints: Current Challenges, pages 63-82, 2018.

[8] H. Fujioka. A discrete-time approach to stability analysis of systems with aperiodic sample-and-hold devices. IEEE Transactions on Automatic Control, 54(10):2440-2445, Oct 2009.

[9] H. Fujioka. Stability analysis of systems with aperiodic sample-and-hold devices. Automatica, 45(3):771 - 775, 2009.

[10] W. P. M. H. Heemels, A. R. Teel, N. van de Wouw, and D. Nesic. Networked control systems with communication constraints: Tradeoffs between transmission intervals, delays and performance. IEEE
Transactions on Automatic Control, 55(8):17811796, Aug 2010.

[11] J. P. Hespanha, P. Naghshtabrizi, and Y. Xu. A survey of recent results in networked control systems. Proceedings of the IEEE, 95(1):138-162, Jan 2007.

[12] L. Hetel, C. Fiter, H. Omran, A. Seuret, E. Fridman, J.-P. Richard, and S. I. Niculescu. Recent developments on the stability of systems with aperiodic sampling: An overview. Automatica, 76:309$335,2017$.

[13] C.-Y. Kao and B. Lincoln. Simple stability criteria for systems with time-varying delays. Automatica, 40(8):1429 - 1434, 2004.

[14] I. Karafyllis and C. Kravaris. Global stability results for systems under sampled-data control. In 2007 European Control Conference (ECC), pages 5761-5768, July 2007.

[15] I. Karafyllis and M. Krstic. Nonlinear stabilization under sampled and delayed measurements, and with inputs subject to delay and zero-order hold. IEEE Transactions on Automatic Control, 57(5):1141-1154, May 2012.

[16] K. Liu, E. Fridman, and L. Hetel. Network-based control via a novel analysis of hybrid systems with time-varying delays. In 2012 IEEE 51st IEEE Conference on Decision and Control $(C D C)$, pages 3886-3891, Dec 2012.

[17] K. Liu, V. Suplin, and E. Fridman. Stability of linear systems with general sawtooth delay. IMA Journal of Mathematical Control and Information, 27(4):419, 2010.

[18] M. Mattioni, S. Monaco, and D. Normand-Cyrot. Nonlinear discrete-time systems with delayed control: A reduction. Systems \& Control Letters, 114:31 $-37,2018$.

[19] F. Mazenc, M. Malisoff, and T. N. Dinh. Robustness of nonlinear systems with respect to delay and sampling of the controls. Automatica, 49(6):19251931, 2013.

[20] L. Mirkin. Some remarks on the use of time-varying delay to model sample-and-hold circuits. IEEE Transactions on Automatic Control, 52(6):11091112, June 2007.

[21] D. Nesic, A. R. Teel, and D. Carnevale. Explicit computation of the sampling period in emulation of controllers for nonlinear sampled-data systems. IEEE Transactions on Automatic Control, 54(3):619-624, March 2009.

[22] D. Nešić, A. R. Teel, and D. Carnevale. Explicit computation of the sampling period in emulation of controllers for nonlinear sampled-data systems. IEEE Transactions on Automatic Control, 54(3):619-624, March 2009.

[23] H. Omran, L. Hetel, M. Petreczky, J. P. Richard, and F. Lamnabhi-Lagarrigue. Stability analysis of some classes of input-affine nonlinear systems with aperiodic sampled-data control. Automatica, 70:266 - 274, 2016.

[24] A. Papachristodoulou, J. Anderson, G. Valmorbida, 
S. Prajna, P. Seiler, and P. A. Parrilo. SOSTOOLS: Sum of squares optimization toolbox for MATLAB, 2013.

[25] P. Pepe, G. Pola, and M. D. D. Benedetto. On Lyapunov Krasovskii characterizations of stability notions for discrete-time systems with uncertain timevarying time delays. IEEE Transactions on Automatic Control, 63(6):1603-1617, June 2018.

[26] Ilia G. Polushin and Horacio J. Marquez. Multirate versions of sampled-data stabilization of nonlinear systems. Automatica, 40(6):1035 - 1041, 2004.

[27] R. Postoyan, P. Tabuada, D. Nešić, and A. Anta. A framework for the event-triggered stabilization of nonlinear systems. IEEE Transactions on Automatic Control, 60(4):982-996, April 2015.

[28] A. Seuret. A novel stability analysis of linear systems under asynchronous samplings. Automatica, 48(1):177 - 182, 2012.

[29] A. Seuret and F. Gouaisbaut. Jensen's and Wirtinger's inequalities for time-delay systems. IFAC Proceedings Volumes, 46(3):343 - 348, 2013. 11th Workshop on Time-Delay Systems.

[30] J. Thomas, L. Hetel, C. Fiter, N. van de Wouw, and J. Richard. $L_{2}$-stability criterion for systems with decentralized asynchronous controllers. In 2018 IEEE Conference on Decision and Control (CDC), pages 6638-6643, Dec 2018.

[31] N. van de Wouw, P. Naghshtabrizi, M. B. G. Cloosterman, and J. P. Hespanha. Tracking control for sampled-data systems with uncertain time-varying sampling intervals and delays. International Journal of Robust and Nonlinear Control, 20(4):387-411, 2010 .

[32] N. van. de. Wouw, D. Nešić, and W.P.M.H. Heemels. A discrete-time framework for stability analysis of nonlinear networked control systems. Automatica, 48(6):1144 - 1153, 2012.

[33] Jan. C. Willems. Dissipative dynamical systems. European Journal of Control, 13(2):134-151, 2007.

[34] F. Xiao, Y. Shi, and W. Ren. Robustness analysis of asynchronous sampled-data multiagent networks with time-varying delays. IEEE Transactions on Automatic Control, 63(7):2145-2152, July 2018.

[35] W. Zhang, M. S. Branicky, and S. M. Phillips. Stability of networked control systems. IEEE Control Systems Magazine, 21(1):84-99, Feb 2001.

[36] Wei Zhang. Stability Analysis of Networked Control Systems. PhD thesis, 2001.

\section{A Proof of Theorem 1}

Let us first upper-bound the response $x(t)$ for all $t \geq a_{0}$. Consider the function

$$
W(t)=e^{\alpha\left(t-a_{0}\right)} V(x(t))-\int_{a_{0}}^{t} \mathcal{S}(\theta, y(\theta), \omega(\theta)) d \theta, \forall t \geq a_{0},
$$

From condition (13), we have $\dot{W}(t) \leq 0$, for all $t \geq a_{0}$ and therefore $W(t) \leq W\left(a_{0}\right)$, for all $t \geq a_{0}$, which can be stated as $e^{\alpha\left(t-a_{0}\right)} V(x(t))-\int_{a_{0}}^{t} \mathcal{S}(\theta, y(\theta), \omega(\theta)) d \theta \leq$ $V\left(x\left(a_{0}\right)\right)$. Therefore, for all $t \geq a_{0}$, we obtain

$$
\begin{aligned}
V(x(t)) \leq & e^{-\alpha\left(t-a_{0}\right)}\left[-\int_{0}^{a_{0}} \mathcal{S}(\theta, y(\theta), \omega(\theta)) d \theta\right. \\
& \left.+\int_{0}^{t} \mathcal{S}(\theta, y(\theta), \omega(\theta)) d \theta+V\left(x\left(a_{0}\right)\right)\right]
\end{aligned}
$$

and by using (9), for all $\theta \geq 0$, we have

$$
\begin{aligned}
V(x(t)) \leq e^{-\alpha\left(t-a_{0}\right)}[ & {\left[-\int_{0}^{a_{0}} \mathcal{S}(\theta, y(\theta), \omega(\theta)) d \theta\right.} \\
+ & \left.+V\left(x\left(a_{0}\right)\right)\right] .
\end{aligned}
$$

By integrating condition (12) for all $t \in\left[0, a_{0}\right)$, we have

$$
V\left(x\left(a_{0}\right)\right) \geq e^{\lambda\left(a_{0}-t\right)} V(x(t)), \forall t \in\left[0, a_{0}\right) .
$$

Then, by integrating condition (11) and using (A.4) for all $t \in\left[0, a_{0}\right)$, we have $-\int_{0}^{a_{0}} \mathcal{S}(\theta, y(\theta), \omega(\theta)) d \theta \leq$ $\rho \int_{0}^{a_{0}} V(x(\theta)) d \theta \leq \rho \int_{0}^{a_{0}} e^{\lambda\left(\theta-a_{0}\right)} V\left(x\left(a_{0}\right)\right) d \theta=$ $\eta V\left(x\left(a_{0}\right)\right)$, where $\eta:=\left\{\begin{array}{l}\frac{\rho e^{-\lambda a_{0}}}{\lambda}\left(e^{\lambda a_{0}}-1\right), \text { if } \lambda \neq 0, \\ \rho a_{0}, \text { if } \lambda=0 .\end{array}\right.$

Consequently, from (A.3),

$$
\begin{aligned}
V(x(t)) & \leq e^{-\alpha\left(t-a_{0}\right)}(1+\eta) V\left(x\left(a_{0}\right)\right), \\
& =e^{-\alpha\left(t-a_{0}\right)} \mathcal{C} V\left(x\left(a_{0}\right)\right), \forall t \geq a_{0}
\end{aligned}
$$

with $\mathcal{C}:=\eta+1>1$. Then, from (10), we obtain for all $t \geq a_{0}, c_{1}\|x(t)\|^{q} \leq V(x(t)) \leq e^{-\alpha\left(t-a_{0}\right)} \mathcal{C} V\left(x\left(a_{0}\right)\right) \leq$ $e^{-\alpha\left(t-a_{0}\right)} \mathcal{C} c_{2}\left\|x\left(a_{0}\right)\right\|^{q}$, and thus

$$
\|x(t)\| \leq \sqrt[q]{\mathcal{C} \frac{c_{2}}{c_{1}}} e^{\frac{-\alpha}{q}\left(t-a_{0}\right)}\left\|x\left(a_{0}\right)\right\|, \forall t \geq a_{0} .
$$

Now, let us analyse the response in the interval $t \in$ $\left[0, a_{0}\right)$. Using the definition of system $\Sigma$ in (7) for all $t \in\left[0, a_{0}\right)$, we have $\dot{x}(t)=\bar{f}_{0}(x(t))$, where $\bar{f}_{0}$ is globally Lipschitz continuous with some constant $k_{0}$ and $\bar{f}_{0}(0)=$ 0 . Hence, we have that $x(t)-x(0)=\int_{0}^{t} \bar{f}_{0}(x(s)) d s$, implying, using the Triangular Inequality, that

$$
\|x(t)\| \leq\|x(0)\|+\int_{0}^{t}\left\|\bar{f}_{0}(x(s))\right\| d s .
$$

Since $\bar{f}_{0}$ is Lipschitz continuous and $\bar{f}_{0}(0)=0$, we have $\left\|\bar{f}_{0}(x(s))\right\|=\left\|\bar{f}_{0}(x(s))-\bar{f}_{0}(0)\right\| \leq k_{0}\|x(s)-0\|=$ $k_{0}\|x(s)\|$. Consequently, (A.7) leads to $\|x(t)\| \leq$ $\|x(0)\|+k_{0} \int_{0}^{t}\|x(s)\| d s$. By virtue of Gronwall's inequality, we obtain

$$
\|x(t)\| \leq\|x(0)\| e^{k_{0} t}, \forall t \in\left[0, a_{0}\right),
$$


implying $\left\|x\left(a_{0}\right)\right\| \leq e^{k_{0} a_{0}}\|x(0)\|$. From (A.6), we obtain

$$
\|x(t)\| \leq \sqrt[q]{\mathcal{C} \frac{c_{2}}{c_{1}}} e^{\frac{-\alpha}{q}\left(t-a_{0}\right)} e^{k_{0} a_{0}}\|x(0)\|, \forall t \geq a_{0} .
$$

Additionally, for all $t \in\left[0, a_{0}\right)$, we can upper-bound inequality (A.8) by $\|x(t)\| \leq e^{k_{0} t}\|x(0)\| \leq e^{k_{0} a_{0}}\|x(0)\| \leq$ $\sqrt[q]{\mathcal{C} \frac{c_{2}}{c_{1}}} e^{\frac{-\alpha}{q}\left(t-a_{0}\right)} e^{k_{0} a_{0}}\|x(0)\|, \forall t \in\left[0, a_{0}\right)$, since $\mathcal{C}>1$ $($ see $(\mathrm{A} .5)), c_{2} \geq c_{1}$ (see $\left.(10)\right)$, and $\frac{-\alpha}{q}\left(t-a_{0}\right) \geq$ 0 . Consequently, using (A.9), we obtain $\|x(t)\| \leq$ $\sqrt[q]{\mathcal{C} \frac{c_{2}}{c_{1}}} e^{\frac{-\alpha}{q}\left(t-a_{0}\right)} e^{k_{0} a_{0}}\|x(0)\|=\delta e^{-\frac{\alpha t}{q}}\|x(0)\|$, for all $t \geq 0$, with $\delta:=e^{\left(k_{0}+\frac{\alpha}{q}\right) a_{0}} \sqrt[q]{\mathcal{C} \frac{c_{2}}{c_{1}}}$, thereby implying that the system $\Sigma-\Delta$ is exponentially stable with a decay-rate of at least $\alpha / q$.

\section{B Proof of Lemma 2}

(1) For all $t \in\left[0, a_{0}\right)$ : As per the definition of $e(t)$ in (14) and $\Delta$ in (15), we have $e(t)=0=\left(\Delta \dot{u}_{c}\right)(t), \forall t \in\left[0, a_{0}\right)$. (2) For all $t \in\left[a_{k}, a_{k+1}\right), k \in \mathbb{N}$ : We have $e(t)=\kappa\left(x_{p}\left(s_{k}\right)\right)-$

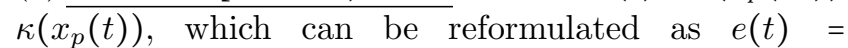
$-\int_{s_{k}}^{t} \frac{d}{d s} \kappa\left(x_{p}(s)\right) d s=-\int_{s_{k}}^{t} \dot{u}_{c}(s) d s$. Therefore, using the definition of $\Delta$ in (15), it can be concluded that indeed $e(t)=\left(\Delta \dot{u}_{c}\right)(t)$.

\section{Proof of Lemma 3}

Consider the system $\mathcal{P}$ in (1), (2), (4)-(6). Moreover, consider the following notational relations:

$$
y(t)=\dot{u}_{c}(t)
$$

with $\dot{u}_{c}(t)$ given by $(16)$, and $\omega(t)=e(t)$, with $e(t)$ defined by (14). By virtue of Lemma 2, we have, $\omega(t)=$ $e(t)=\left(\Delta \dot{u}_{c}\right)(t)=(\Delta y)(t), \forall t \geq 0$.

(1) For all $t \in\left[0, a_{0}\right)$ : As per the definition of system $\mathcal{P}$, we have

$$
\dot{x}_{p}(t)=f\left(x_{p}(t)\right)
$$

and using (16),

$$
y(t)=\dot{u}_{c}(t)=\frac{d}{d t} \kappa\left(x_{p}(t)\right)=\frac{\partial \kappa\left(x_{p}(t)\right)}{\partial x_{p}} f\left(x_{p}(t)\right) .
$$

Using (17), (C.2) and (C.3) this is equivalent to $\dot{x}_{p}(t)=\bar{f}_{0}\left(x_{p}(t)\right), y(t)=\bar{h}_{0}\left(x_{p}(t)\right)$. This expresses the dynamics of system $\Sigma$ for $t \in\left[0, a_{0}\right)$, given by (7), with $\vec{f}_{0}$ and $\bar{h}_{0}$ as defined in (17), i.e, for all $t \in\left[0, a_{0}\right)$, $x(t)=x_{p}(t)$. Additionally, using $\bar{f}_{0}(x)=f(x)$, it can be concluded from the definition of system $\mathcal{P}$ that the function $\bar{f}_{0}$ is globally Lipschitz continuous with $\bar{f}_{0}(0)=0$.

(2) For all $t \in\left[a_{k}, a_{k+1}\right), k \in \mathbb{N}$ : The dynamics of system $\overline{\mathcal{P}}$ is given by $\dot{x}_{p}(t)=f\left(x_{p}(t)\right)+g\left(x_{p}(t)\right) u(t)=$ $f\left(x_{p}(t)\right)+g\left(x_{p}(t)\right) \kappa\left(x_{p}\left(s_{k}\right)\right)=f\left(x_{p}(t)\right)+$ $g\left(x_{p}(t)\right) \kappa\left(x_{p}(t)\right)+g\left(x_{p}(t)\right)\left[\kappa\left(x_{p}\left(s_{k}\right)\right)-\kappa\left(x_{p}(t)\right)\right]$. Using (17), and recalling the definition of $e(t)$ in (14), we obtain $\dot{x}_{p}(t)=\bar{f}\left(x_{p}(t)\right)+\bar{g}\left(x_{p}(t)\right) e(t)$. This is equivalent to the dynamics of system $\Sigma$ for $t \geq a_{0}$, given by (7), with $\omega(t)=e(t)$ and the functions $\bar{f}$ and $\bar{g}$ defined by (17), i.e., for all $t \geq a_{0}$, we have $x=x_{p}$.

Additionally, from (C.1) and (16) we have, $y(t)=$ $\frac{d}{d t} \kappa\left(x_{p}(t)\right)=\frac{\partial \kappa\left(x_{p}(t)\right)}{\partial x_{p}}\left(\bar{f}\left(x_{p}(t)\right)+\bar{g}\left(x_{p}(t)\right) e(t)\right)$. Once again, using notation (17) and $e(t)=\omega(t)$, we have, $y(t)=\bar{h}\left(x_{p}(t)\right)+\bar{l}\left(x_{p}(t)\right) \omega(t)$, which is the same as $y$ defined in (7), for $t \geq a_{0}$, since we have already shown $x=x_{p}$. Therefore, it can be seen that system $\mathcal{P}$ can be expressed as the feedback interconnection $\Sigma-\Delta$, with the functions $\bar{f}_{0}, \bar{h}_{0}, \bar{f}, \bar{g}, \bar{h}$, and $\bar{l}$ defined by (17).

\section{Proof of Lemma 4}

(1) For $t \in\left[0, a_{0}\right)$ : Using the definition of $\Delta$ in (15), we have $(\Delta z)(\theta)=0$, for all $\theta \in[0, t)$ and clearly (18) holds in this case since $S(\theta, z(\theta),(\Delta z)(\theta))=$ $-\gamma^{2} z^{T}(\theta) R z(\theta) \leq 0$.

(2) For $t \geq a_{0}$ : Let $w(t)$ denote

$$
w(t)=(\Delta z)(t)=-\int_{s_{k}}^{t} z(\zeta) d \zeta, \forall t \in\left[a_{k}, a_{k+1}\right), k \in \mathbb{N} .
$$

Using Jensen's inequality, we obtain $w^{T}(t) R w(t) \leq(t-$ $\left.s_{k}\right) \int_{s_{k}}^{t} z^{T}(\zeta) R z(\zeta) d \zeta \leq(\bar{h}+\bar{\tau}) \int_{s_{k}}^{t} z^{T}(\zeta) R z(\zeta) d \zeta$. Using the change of variable $s=\zeta-t$, we obtain $w^{T}(t) R w(t) \leq$ $(\bar{h}+\bar{\tau}) \int_{s_{k}-t}^{0} z^{T}(t+s) R z(t+s) d s \leq(\bar{h}+\bar{\tau}) \int_{-(\bar{h}+\bar{\tau})}^{0} z^{T}(t+$ $s) R z(t+s) d s$. Therefore, $\int_{a_{0}}^{t} e^{\alpha\left(\theta-a_{0}\right)} w^{T}(\theta) R w(\theta) d \theta \leq$ $(\bar{h}+\bar{\tau}) \int_{a_{0}}^{t} e^{\alpha\left(\theta-a_{0}\right)}\left(\int_{-(\bar{h}+\bar{\tau})}^{0} z^{T}(\theta+s) R z(\theta+s) d s\right) d \theta$. Substituting $u=\theta+s$, we have that

$$
\begin{aligned}
& \int_{a_{0}}^{t} e^{\alpha\left(\theta-a_{0}\right)} w^{T}(\theta) R w(\theta) d \theta \\
& \leq(\bar{h}+\bar{\tau}) \int_{-(\bar{h}+\bar{\tau})}^{0}\left(\int_{a_{0}+s}^{t+s} e^{\alpha\left(u-s-a_{0}\right)} z^{T}(u) R z(u) d u\right) d s .
\end{aligned}
$$

Since the inner integral in the right-hand side of the inequality in (D.2) is always positive, we can upper bound the left-hand side in (D.2) using the limits of $s$ and obtain $\int_{a_{0}}^{t} e^{\alpha\left(\theta-a_{0}\right)} w^{T}(\theta) R w(\theta) d \theta \leq(\bar{h}+$ $\bar{\tau}) \int_{-(\bar{h}+\bar{\tau})}^{0}\left(\int_{a_{0}-(\bar{h}+\bar{\tau})}^{t+0} e^{\alpha\left(u+(\bar{h}+\bar{\tau})-a_{0}\right)} z^{T}(u) R z(u) d u\right) d s \leq$ $(\bar{h}+\bar{\tau}) e^{\alpha(\bar{h}+\bar{\tau})} \int_{-(\bar{h}+\bar{\tau})}^{0}\left(\int_{0}^{t} e^{\alpha\left(u-a_{0}\right)} z^{T}(u) R z(u) d u\right) d s=$ $(\bar{h}+\bar{\tau})^{2} e^{\alpha(\bar{h}+\bar{\tau})} \int_{0}^{t} e^{\alpha\left(u-a_{0}\right)} z^{T}(u) R z(u) d u$. As per definition (15), we have $w(t)=0$ for all $0 \leq t<$ $a_{0}$ and, consequently, $\int_{0}^{t} e^{\alpha\left(\theta-a_{0}\right)} w^{T}(\theta) R w(\theta) d \theta=$ $\int_{a_{0}}^{t} e^{\alpha\left(\theta-a_{0}\right)} w^{T}(\theta) R w(\theta) d \theta \leq(\bar{h}+\bar{\tau})^{2} e^{\alpha(\bar{h}+\bar{\tau})} \int_{0}^{t} e^{\alpha\left(u-a_{0}\right)}$ $z^{T}(u) R z(u) d u$. Hence, using the definition of $w(t)$ in (D.1), we have that $\int_{0}^{t} e^{\alpha\left(\theta-a_{0}\right)}\left((\Delta z)^{T}(\theta) R(\Delta z)(\theta)-\right.$ $\left.(\bar{h}+\bar{\tau})^{2} e^{\alpha(\bar{h}+\bar{\tau})} z^{T}(\theta) R z(\theta)\right) d \theta \leq 0$, which proves the integral inequality (18), thereby concluding the proof. 


\section{E $\quad$ Proof of Theorem 6}

Comparing the sampled-data systems $\mathcal{P}_{L}$ and $\mathcal{P}$, we have, $f(x(t)):=A x(t), g(x(t)):=B, \kappa\left(x\left(s_{k}\right)\right):=$ $K x\left(s_{k}\right)$. Hence, the sampling and delay induced error is given by $e(t)=\left\{\begin{array}{l}0, \forall t \in\left[0, a_{0}\right), \\ K x\left(s_{k}\right)-K x(t), \forall t \in\left[a_{k}, a_{k+1}\right), k \in \mathbb{N},\end{array}\right.$ thereby implying that using the operator $\Delta$ defined in (15), we can state $e=\Delta(K \dot{x})$. Using the inequality in (22), we proceed to prove that the assumptions introduced in Theorem 1 will hold for $V(x)=x^{T} P x$ and $\mathcal{S}(t, y(t), w(t))$ defined by (19). For the LTI system $\mathcal{P}_{L}$, the functions given in (17) are given by $\bar{f}_{0}(x(t)):=$ $A x(t), \bar{h}_{0}(x(t)):=K A x(t), \bar{f}(x(t)):=\bar{A} x(t), \bar{g}(x(t)):=$ $B, h(x(t)):=K \bar{A} x(t)$, and $\bar{l}(x(t)):=K B$, where $\bar{A}=(A+B K)$.

(1) Satisfying Assumption 1, i.e., (9): By virtue of Lemma 4, we can see that the supply function $\mathcal{S}(t, y(t), w(t))$ defined by (19) satisfies assumption (9) in Theorem 1, i.e., $\int_{0}^{t} \mathcal{S}(\theta, y(\theta),(\Delta y)(\theta)) d \theta \leq 0, \forall t \geq 0$. (2) Satisfying Assumption 2, i.e., (10): With $V(x)=$ $x^{T} P \overline{x, P}=P^{T}>0$ and $x \in \mathbb{R}^{n}$, we have that $\delta_{\min }(P)\|x\|^{2} \leq x^{T} P x \leq \delta_{\max }(P)\|x\|^{2}$, implying Assumption 2 is satisfied with $q=2, c_{1}=\delta_{\min }(P)$ and $c_{2}=\delta_{\max }(P)$.

(3) Satisfying Assumption 3, inequality (11): Consider the function $\mathcal{S}(t, y(t), w(t))$ defined by (19). For all $t \in\left[0, a_{0}\right)$, since $y(t)=\bar{h}_{0}(x(t))=K A x(t)$ and $\omega(t)=0$, we have that for all $t \in\left[0, a_{0}\right),-\mathcal{S}(t, y(t), \omega(t))=$ $-\mathcal{S}\left(t, \bar{h}_{0}(x(t)), 0\right)=e^{\alpha\left(t-a_{0}\right)} \gamma^{2} x^{T}(t)(K A)^{T} R(K A) x(t)$ $\leq \max _{\theta \in\left[0, a_{0}\right]}\left\{\delta_{\max }\left[e^{\alpha\left(\theta-a_{0}\right)} \gamma^{2}(K A)^{T} R(K A)\right]\right\}\|x(t)\|^{2}$ $\leq \rho V(x(t))$, with $\rho=\frac{\delta_{\max }\left(\gamma^{2}(K A)^{T} R(K A)\right)}{\delta_{\min }(P)}$.

(4) Satisfying Assumption 3, inequality (12): We have $V(x(t))=x(t)^{T} P x(t)$ for all $t \geq 0$. For all $t \in\left[0, a_{0}\right)$, $\dot{x}(t)=\bar{f}_{0}(x(t))=A x(t), \dot{V}(x(t))=x(t)^{T}\left[A^{T} P+P A\right]$ $x(t) \geq \frac{\delta_{\min }\left(A^{T} P+P A\right)}{\delta_{\max }(P)} V(x(t))$. Therefore, inequality

(12) is satisfied for any $\lambda \leq \frac{\delta_{\min }\left(A^{T} P+P A\right)}{\delta_{\max }(P)}$.

(5) Satisfying Assumption 3, inequality (13): Consider the function $W(t)=\dot{V}(x(t))+\alpha V(x(t))-e^{-\alpha\left(t-a_{0}\right)}$ $\mathcal{S}(t, y(t), e(t))$, defined for all $t \geq a_{0}$ with $V(x)=x^{T} P x$, and the function $\mathcal{S}(t, y(t), e(t))$ defined by (19). Clearly, inequality (13) in Assumption 3 holds if $W(t) \leq 0$, for all $t \geq a_{0}$. We have, $\mathcal{S}(t, y(t), e(t))=$ $e^{\alpha\left(t-a_{0}\right)}\left[\begin{array}{l}y(t) \\ e(t)\end{array}\right]^{T}\left[\begin{array}{cc}-\gamma^{2} R & 0 \\ 0 & R\end{array}\right]\left[\begin{array}{l}y(t) \\ e(t)\end{array}\right]=e^{\alpha\left(t-a_{0}\right)}\left[\begin{array}{l}x(t) \\ e(t)\end{array}\right]^{T}$ $N\left[\begin{array}{l}x(t) \\ e(t)\end{array}\right]$, where $N=\left[\begin{array}{cc}K \bar{A} & K B \\ 0 & I\end{array}\right]^{T}\left[\begin{array}{cc}-\gamma^{2} R & 0 \\ 0 & R\end{array}\right]\left[\begin{array}{cc}K \bar{A} & K B \\ 0 & I\end{array}\right]$. Therefore, we have that for all $t \geq a_{0}$, $W(t)=\dot{V}(x(t))+\alpha V(x(t))-e^{-\alpha\left(t-a_{0}\right)} \mathcal{S}(t, y(t), e(t))=$ $\left[\begin{array}{l}x(t) \\ e(t)\end{array}\right]^{T}\left\{\left[\begin{array}{cc}\bar{A}^{T} P+P \bar{A} & P B \\ B^{T} P & 0\end{array}\right]+\alpha\left[\begin{array}{ll}P & 0 \\ 0 & 0\end{array}\right]-N\right\}\left[\begin{array}{l}x(t) \\ e(t)\end{array}\right]=$ $\left[\begin{array}{l}x(t) \\ e(t)\end{array}\right]^{T} \Gamma\left[\begin{array}{l}x(t) \\ e(t)\end{array}\right]$, with $\Gamma:=\left[\begin{array}{cc}\bar{A}^{T} P+P \bar{A}+\alpha P & P B \\ B^{T} P & 0\end{array}\right]-N$. Substituting $N$ in the expression for $\Gamma$ gives $\Gamma=$ $\left[\begin{array}{cc}\bar{A}^{T} P+P \bar{A}+\alpha P & P B \\ B^{T} P & 0\end{array}\right]-N=\left[\begin{array}{cc}\bar{A}^{T} P+P \bar{A}+\alpha P & P B \\ B^{T} P & 0\end{array}\right]+$ $\left[\begin{array}{cc}K \bar{A} & K B \\ 0 & I\end{array}\right]^{T}\left[\begin{array}{cc}\gamma^{2} R & 0 \\ 0 & -R\end{array}\right]\left[\begin{array}{cc}K \bar{A} & K B \\ 0 & I\end{array}\right]$. A sufficient condition for $W(t) \leq 0$ for all $t \geq a_{0}$ will therefore be given by $\Gamma \leq 0$, which is guaranteed by (22). Consequently, we have proved that inequality (13) in Assumption 3 is satisfied for the chosen storage and supply functions.

We have shown that all the assumptions of Theorem 1 hold for $V(x)=x^{T} P x$ and $\mathcal{S}(t, y(t), e(t))$ defined by (19) and, therefore, the exponential stability of system $\mathcal{P}$ is guaranteed with a decay rate greater than or equal to $\alpha / 2$.

\section{F $\quad$ Proof of Lemma 7}

(1) Expressing $e_{s}$ using $\Delta_{s}$ : Recalling the definition of $e_{s}(t)$ in (24), and by using the operator definition for $\Delta_{s}$ in (27), we can state using (16) that for all $t \in\left[0, s_{0}\right), e_{s}(t)=0=\left(\Delta_{s} \dot{u}_{c}\right)(t)$. Similarly, for all $t \in\left[s_{k}, s_{k+1}\right), k \in \mathbb{N}, e_{s}(t)=\kappa\left(x_{p}\left(s_{k}\right)\right)-\kappa\left(x_{p}(t)\right)=$ $-\int_{s_{k}}^{t} \frac{d}{d s} \kappa\left(x_{p}(s)\right) d s=-\int_{s_{k}}^{t} \dot{u}_{c}(s) d s=\left(\Delta_{s} \dot{u}_{c}\right)(t)$. Hence, we have $e_{s}(t)=\left(\Delta_{s} \dot{u}_{c}\right)(t), \forall t \geq 0$.

(2) Expressing $e_{d}$ using $\Delta_{d}$ : In a similar manner, using the definition of $e_{d}(t)$ in (25) and the operator definition for $\Delta_{d}$ defined in (28), we have, for all $t \in\left[0, a_{0}\right) \cup$ $\left[a_{k-1}, s_{k}\right)_{k \in \mathbb{N}^{*}}, e_{d}(t)=0=\left(\Delta_{d} \dot{u}_{c}\right)(t)$. Similarly, for all $t \in\left[s_{k}, a_{k}\right), k \in \mathbb{N}^{\star}, e_{d}(t)=\kappa\left(x_{p}\left(s_{k-1}\right)\right)-\kappa\left(x_{p}\left(s_{k}\right)\right)=$ $-\int_{s_{k-1}}^{s_{k}} \frac{d}{d s} \kappa\left(x_{p}(s)\right) d s=-\int_{s_{k-1}}^{s_{k}} \dot{u}_{c}(s) d s=\left(\Delta_{d} \dot{u}_{c}\right)(t)$. Hence, we obtain $e_{d}(t)=\left(\Delta_{d} \dot{u}_{c}\right)(t), \forall t \geq 0$.

\section{G Proof of Lemma 8}

Consider system $\mathcal{P}$, the notations $y(t)=\left[y_{1}(t) y_{2}(t)\right]^{T}=$ $\left[\dot{u}_{c}(t) \dot{u}_{c}(t)\right]^{T}$, with $\dot{u}_{c}$ defined by $(16)$, and $\omega(t)=$ $\left[e_{s}(t) e_{d}(t)\right]^{T}$, with $e_{s}(t)$ and $e_{d}(t)$ given by $(24)$ and (25), respectively. By virtue of Lemma 7 , we have

$$
\omega(t)=\left[\begin{array}{l}
e_{s}(t) \\
e_{d}(t)
\end{array}\right]=\left[\begin{array}{l}
\left(\Delta_{s} \dot{u}_{c}\right)(t) \\
\left(\Delta_{d} \dot{u}_{c}\right)(t)
\end{array}\right]=(\Delta y)(t), \forall t \geq 0
$$

with $\Delta_{s}$ and $\Delta_{d}$ given in (27) and (28), respectively. In order to establish the equivalence between system $\mathcal{P}$ 
and the feedback interconnection $\Sigma-\Delta$, we begin by reformulating the dynamics of system $\mathcal{P}$ for all $t \in\left[0, a_{0}\right)$, $t \in\left[a_{k}, s_{k+1}\right)_{k \in \mathbb{N}}$, and $t \in\left[s_{k+1}, a_{k+1}\right)_{k \in \mathbb{N}}$, i.e., for all $t \geq 0$. (1) For all $t \in\left[0, a_{0}\right)$ : Consider the system $\mathcal{P}$. We have that $\dot{x}_{p}(t)=f\left(x_{p}(t)\right)$, and using (16), $y_{1}(t)=y_{2}(t)=$ $\dot{u}_{c}(t)=\frac{d}{d t} \kappa\left(x_{p}(t)\right)=\frac{\partial \kappa\left(x_{p}(t)\right)}{\partial x_{p}} \dot{x}_{p}(t)=\frac{\partial \kappa\left(x_{p}(t)\right)}{\partial x_{p}} f\left(x_{p}(t)\right)$. Therefore, using the notation in (30), we obtain $\dot{x}_{p}(t)=\bar{f}_{0}\left(x_{p}(t)\right), y(t)=\left[y_{1}(t) y_{2}(t)\right]^{T}=\bar{h}_{0}\left(x_{p}(t)\right)$. Note that this is the dynamics of system $\sum$ for $t \in\left[0, a_{0}\right)$, given by $(7)$, with the functions $\bar{f}_{0}$ and $\bar{h}_{0}$ as defined in (30), i.e., for all $t \in\left[0, a_{0}\right), x(t)=x_{p}(t)$. Additionally, as per the notation in $(17)$, since $\bar{f}_{0}(x)=f(x)$, it can be concluded from the definition of system $\mathcal{P}$ that the function $\bar{f}_{0}$ is globally Lipschitz continuous with $\bar{f}_{0}(0)=0$.

(2) For all $t \in\left[a_{k}, s_{k+1}\right), k \in \mathbb{N}$ : The dynamics of system $\overline{\mathcal{P}}$ is given by $\dot{x}_{p}(t)=f\left(x_{p}(t)\right)+g\left(x_{p}(t)\right) u(t)=$ $f\left(x_{p}(t)\right)+g\left(x_{p}(t)\right) \kappa\left(x_{p}\left(s_{k}\right)\right)=f\left(x_{p}(t)\right)+g\left(x_{p}(t)\right)$ $\kappa\left(x_{p}(t)\right)+g\left(x_{p}(t)\right)\left[\kappa\left(x_{p}\left(s_{k}\right)\right)-\kappa\left(x_{p}(t)\right)\right]$. Using the definitions of sampling and delay induced errors in (24) and (25), respectively, we have $e_{s}(t)=$ $\kappa\left(x_{p}\left(s_{k}\right)\right)-\kappa\left(x_{p}(t)\right), \forall t \in\left[a_{k}, s_{k+1}\right)$, and $e_{d}(t)=0$ for all $t \in\left[a_{k}, s_{k+1}\right)$. Therefore, we can reformulate the dynamics of system $\mathcal{P}$ for all $t \in\left[a_{k}, s_{k+1}\right)$ as $\dot{x}_{p}(t)=f\left(x_{p}(t)\right)+$ $g\left(x_{p}(t)\right) \kappa\left(x_{p}(t)\right)+g\left(x_{p}(t)\right) e_{s}(t)+g\left(x_{p}(t)\right) e_{d}(t)=$ $f\left(x_{p}(t)\right)+g\left(x_{p}(t)\right) \kappa\left(x_{p}(t)\right)+\left[g\left(x_{p}(t)\right) \quad g\left(x_{p}(t)\right)\right]$ $\left[e_{s}(t) e_{d}(t)\right]^{T}$. Using the notation in (30), this can be written as $\dot{x}_{p}(t)=\bar{f}\left(x_{p}(t)\right)+\bar{g}\left(x_{p}(t)\right) \omega(t)$, with $\omega(t)$ as in (G.1). This is the same as dynamics of system $\Sigma$ for $t \in\left[a_{k}, s_{k+1}\right), k \in \mathbb{N}$, given by $(7)$, with $\omega$ defined in (G.1), and the functions $\bar{f}$ and $\bar{g}$ defined by (30), i.e., for all $t \in\left[a_{k}, s_{k+1}\right)$, with $x=x_{p}$. Additionally, we have $\dot{u}_{c}(t)=\frac{d}{d t} \kappa\left(x_{p}(t)\right)$ and hence,

$$
y_{1}(t)=y_{2}(t)=\frac{\partial \kappa\left(x_{p}(t)\right)}{\partial x_{p}}\left(\bar{f}\left(x_{p}(t)\right)+\bar{g}\left(x_{p}(t)\right) \omega(t)\right) .
$$

Therefore, using the notation in (30) once again, we obtain $y(t)=\left[\begin{array}{ll}y_{1}(t) & y_{2}(t)\end{array}\right]^{T}=\bar{h}\left(x_{p}(t)\right)+\bar{l}\left(x_{p}(t)\right) \omega(t)$, which is the same as $y$ defined in $(7)$, for $t \in\left[a_{k}, s_{k+1}\right)$, with $x=x_{p}$.

(3) For all $t \in\left[s_{k+1}, a_{k+1}\right), k \in \mathbb{N}$ : Once again, we proceed to reformulate the dynamics of system $\mathcal{P}$ given by $\dot{x}_{p}(t)=f\left(x_{p}(t)\right)+g\left(x_{p}(t)\right) u(t)=f\left(x_{p}(t)\right)+$ $g\left(x_{p}(t)\right) \kappa\left(x_{p}\left(s_{k}\right)\right)=f\left(x_{p}(t)\right)+g\left(x_{p}(t)\right) \kappa\left(x_{p}\left(s_{k}\right)\right)+$ $g\left(x_{p}(t)\right) \kappa\left(x_{p}(t)\right)-g\left(x_{p}(t)\right) \kappa\left(x_{p}(t)\right)+g\left(x_{p}(t)\right)$ $\kappa\left(x_{p}\left(s_{k+1}\right)\right)-g\left(x_{p}(t)\right) \kappa\left(x_{p}\left(s_{k+1}\right)\right)=\left(f\left(x_{p}(t)\right)+\right.$ $\left.g\left(x_{p}(t)\right) \kappa\left(x_{p}(t)\right)\right)+g\left(x_{p}(t)\right)\left[\kappa\left(x_{p}\left(s_{k+1}\right)\right)-\kappa\left(x_{p}(t)\right)\right]+$ $g\left(x_{p}(t)\right)\left[\kappa\left(x_{p}\left(s_{k}\right)\right)-\kappa\left(x_{p}\left(s_{k+1}\right)\right)\right]$. Using the definitions in (24) and (25) and considering Hypothesis 2 , we have that $e_{s}(t)=\kappa\left(x_{p}\left(s_{k+1}\right)\right)-\kappa\left(x_{p}(t)\right), \forall t \epsilon$ $\left[s_{k+1}, s_{k+2}\right) \supset\left[s_{k+1}, a_{k+1}\right)$, and $e_{d}(t)=\kappa\left(x_{p}\left(s_{k}\right)\right)-$ $\kappa\left(x_{p}\left(s_{k+1}\right)\right), \forall t \in\left[s_{k+1}, a_{k+1}\right)$. Therefore, using the notation in (30), we can reformulate the dynamics of system $\mathcal{P}$ for all $t \in\left[s_{k+1}, a_{k+1}\right)$ as $\dot{x}_{p}(t)=\bar{f}\left(x_{p}(t)\right)+$ $g\left(x_{p}(t)\right) e_{s}(t)+g\left(x_{p}(t)\right) e_{d}(t)=\bar{f}\left(x_{p}(t)\right)+\bar{g}\left(x_{p}(t)\right) \omega(t)$. This is the same as dynamics of system $\Sigma$ in (7) for all $t \in\left[s_{k+1}, a_{k+1}\right], k \in \mathbb{N}$, with $\omega(t)$ given by (G.1), and the functions $\bar{f}$ and $\bar{g}$ defined by $(30)$, with $x(t)=x_{p}(t)$. Additionally, using the notation $y_{1}(t)=y_{2}(t)=\dot{u}_{c}(t)$, and following the reasoning given in (G.2), we get, $y(t)=$ $\left[y_{1}(t) y_{2}(t)\right]^{T}=\bar{h}\left(x_{p}(t)\right)+\bar{l}\left(x_{p}(t)\right) \omega(t)$, which is the same as $y$ defined in $(7)$, for $t \in\left[s_{k+1}, a_{k+1}\right), k \in \mathbb{N}$, with $x=x_{p}$.

Therefore, system $\mathcal{P}$ can be expressed in the form of the interconnection $\Sigma-\Delta$, with the functions $\bar{f}_{0}, \bar{h}_{0}, \bar{f}, \bar{g}$, $\bar{h}$, and $\bar{l}$ defined by (30).

\section{H Proof of Lemma 9}

(1) For $t \in\left[0, s_{0}\right)$ : As per the definition of $\Delta_{s}$ in (27), we have that $\left(\Delta_{s} v\right)(t)=0, \forall t \in\left[0, s_{0}\right)$. Therefore, for all $\theta \in\left[0, s_{0}\right)$, we have $\mathcal{S}_{s}\left(\theta, v(\theta),\left(\Delta_{s} v\right)(\theta)\right)=$ $-\gamma_{s}^{2} v^{T}(\theta) R_{s} v(\theta)$, implying that indeed

$\int_{0}^{t} \mathcal{S}_{s}\left(\theta, v(\theta),\left(\Delta_{s} v\right)(\theta)\right) d \theta \leq 0$.

(2) For $t \in\left[s_{k}, s_{k+1}\right), k \in \mathbb{N}$ : We have that

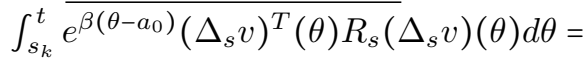

$\int_{s_{k}}^{t} \sqrt{e^{\beta\left(\theta-a_{0}\right)}}\left(\Delta_{s} v\right)^{T}(\theta) R_{s} \sqrt{e^{\beta\left(\theta-a_{0}\right)}}\left(\Delta_{s} v\right)(\theta) d \theta$. Since $\left(\Delta_{s} v\right)\left(s_{k}\right)=0$, by applying Wirtinger's inequality [17], we obtain

$$
\begin{aligned}
& \int_{s_{k}}^{t} e^{\beta\left(\theta-a_{0}\right)}\left(\Delta_{s} v\right)^{T}(\theta) R_{s}\left(\Delta_{s} v\right)(\theta) d \theta \\
& \leq \frac{4\left(t-s_{k}\right)^{2}}{\pi^{2}} \int_{s_{k}}^{t} \frac{d}{d \theta}\left(\sqrt{e^{\beta\left(\theta-a_{0}\right)}}\left(\Delta_{s} v\right)(\theta)\right)^{T} \\
& R_{s} \frac{d}{d \theta}\left(\sqrt{e^{\beta\left(\theta-a_{0}\right)}}\left(\Delta_{s} v\right)(\theta)\right) d \theta
\end{aligned}
$$

with $\frac{d}{d \theta}\left(\sqrt{e^{\beta\left(\theta-a_{0}\right)}}\left(\Delta_{s} v\right)(\theta)\right)=\sqrt{e^{\beta\left(\theta-a_{0}\right)}} \frac{d}{d \theta}\left(\Delta_{s} v\right)(\theta)$ $+\left(\Delta_{s} v\right)(\theta) \frac{\beta}{2} \sqrt{e^{\beta\left(\theta-a_{0}\right)}}$. As per the definition of $\Delta_{s}$ in $(27)$, we have that $\left(\Delta_{s} v\right)(\theta)=-\int_{s_{k}}^{\theta} v(\psi) d \psi, \forall \theta \in$ $\left[s_{k}, s_{k+1}\right), k \in \mathbb{N}$, implying that $\frac{d}{d \theta}\left(\Delta_{s} v\right)(\theta)=-v(\theta)$. Therefore, $\frac{d}{d \theta}\left(\sqrt{e^{\beta\left(\theta-a_{0}\right)}}\left(\Delta_{s} v\right)(\theta)\right)=\sqrt{e^{\beta\left(\theta-a_{0}\right)}}(-v(\theta)+$ $\left.\frac{\beta}{2}\left(\Delta_{s} v\right)(\theta)\right)$, implying that

$\frac{d}{d \theta}\left(\sqrt{e^{\beta\left(\theta-a_{0}\right)}}\left(\Delta_{s} v\right)^{T}(\theta)\right)^{T} R_{s} \frac{d}{d \theta}\left(\sqrt{e^{\beta\left(\theta-a_{0}\right)}}\left(\Delta_{s} v\right)(\theta)\right)=$ $e^{\beta\left(\theta-a_{0}\right)}\left(-v(\theta)+\frac{\beta}{2}\left(\Delta_{s} v\right)(\theta)\right)^{T} R_{s}\left(-v(\theta)+\frac{\beta}{2}\left(\Delta_{s} v\right)(\theta)\right)$ $=e^{\beta\left(\theta-a_{0}\right)}\left(v^{T}(\theta) R_{s} v(\theta)-\frac{\beta}{2} v^{T}(\theta) R_{s}\left(\Delta_{s} v\right)(\theta)-\right.$ $\left.\frac{\beta}{2}\left(\Delta_{s} v\right)^{T}(\theta) R_{s} v(\theta)+\frac{\beta^{2}}{4}\left(\Delta_{s} v\right)^{T}(\theta) R_{s}\left(\Delta_{s} v\right)(\theta)\right)$. Hence,

$$
\begin{gathered}
\frac{d}{d \theta}\left(\sqrt{e^{\beta\left(\theta-a_{0}\right)}}\left(\Delta_{s} v\right)^{T}(\theta)\right)^{T} R_{s} \frac{d}{d \theta}\left(\sqrt{e^{\beta\left(\theta-a_{0}\right)}}\left(\Delta_{s} v\right)(\theta)\right) \\
=e^{\beta\left(\theta-a_{0}\right)} \xi\left(v(\theta),\left(\Delta_{s} v\right)(\theta)\right)
\end{gathered}
$$


where

$$
\begin{aligned}
\xi & \left(v(\theta),\left(\Delta_{s} v\right)(\theta)\right):=\left(v^{T}(\theta) R_{s} v(\theta)\right. \\
& -\frac{\beta}{2} v^{T}(\theta) R_{s}\left(\Delta_{s} v\right)(\theta)-\frac{\beta}{2}\left(\Delta_{s} v\right)^{T}(\theta) R_{s} v(\theta) \\
& \left.+\frac{\beta^{2}}{4}\left(\Delta_{s} v\right)^{T}(\theta) R_{s}\left(\Delta_{s} v\right)(\theta)\right) .
\end{aligned}
$$

Substituting (H.2) into inequality (H.1), we have for all $t \in\left[s_{k}, s_{k+1}\right), k \in \mathbb{N}, \int_{s_{k}}^{t} e^{\beta\left(\theta-a_{0}\right)}\left(\Delta_{s} v\right)^{T}(\theta) R_{s}\left(\Delta_{s} v\right)(\theta) d \theta$ $\leq \frac{4 \bar{h}^{2}}{\pi^{2}} \int_{s_{k}}^{t} e^{\beta\left(\theta-a_{0}\right)} \xi\left(v(\theta),\left(\Delta_{s} v\right)(\theta)\right) d \theta$, where we have used that $\left(t-s_{k}\right) \leq \bar{h}$ for all $t \in\left[s_{k}, s_{k+1}\right)$. Now, for any $t \in\left[s_{k}, s_{k+1}\right)$, since $\left(\Delta_{s} v\right)(t)=0, \forall t<s_{0}($ see $(27))$, we can state that $\int_{0}^{t} e^{\beta\left(\theta-a_{0}\right)}\left(\Delta_{s} v\right)^{T}(\theta) R_{s}\left(\Delta_{s} v\right)(\theta) d \theta=$ $\int_{s_{0}}^{t} e^{\beta\left(\theta-a_{0}\right)}\left(\Delta_{s} v\right)^{T}(\theta) R_{s}\left(\Delta_{s} v\right)(\theta) d \theta$ $=\left(\sum_{i=0}^{k-1} \int_{s_{i}}^{s_{i+1}} e^{\beta\left(\theta-a_{0}\right)}\left(\Delta_{s} v\right)^{T}(\theta) R_{s}\left(\Delta_{s} v\right)(\theta) d \theta\right)+$ $\int_{s_{k}}^{t} e^{\beta\left(\theta-a_{0}\right)}\left(\Delta_{s} v\right)^{T}(\theta) R_{s}\left(\Delta_{s} v\right)(\theta) d \theta$ $\leq \frac{4 \bar{h}^{2}}{\pi^{2}}\left[\left(\sum_{i=0}^{k-1} \int_{s_{i}}^{s_{i+1}} e^{\beta\left(\theta-a_{0}\right)} \xi\left(v(\theta),\left(\Delta_{s} v\right)(\theta)\right) d \theta\right)\right.$ $\left.+\int_{s_{k}}^{t} e^{\beta\left(\theta-a_{0}\right)} \xi\left(v(\theta),\left(\Delta_{s} v\right)(\theta)\right) d \theta\right]$. Therefore,

$$
\begin{aligned}
& \int_{0}^{t} e^{\beta\left(\theta-a_{0}\right)}\left(\Delta_{s} v\right)^{T}(\theta) R_{s}\left(\Delta_{s} v\right)(\theta) d \theta \\
& \leq \frac{4 \bar{h}^{2}}{\pi^{2}}\left[\int_{s_{0}}^{t} e^{\beta\left(\theta-a_{0}\right)} \xi\left(v(\theta),\left(\Delta_{s} v\right)(\theta)\right) d \theta\right] .
\end{aligned}
$$

Since $\left(\Delta_{s} v\right)(t)=0$ for all $t<s_{0}$, and $v(t) \in \mathcal{W}^{m_{p}}$, using the definition of $\xi\left(v(\theta),\left(\Delta_{s} v\right)(\theta)\right)$ in (H.3), $\forall t \in\left[0, s_{0}\right)$,

$$
\begin{aligned}
& \frac{4 \bar{h}^{2}}{\pi^{2}} \int_{0}^{s_{0}} e^{\beta\left(\theta-a_{0}\right)} \xi\left(v(\theta),\left(\Delta_{s} v\right)(\theta)\right) d \theta \\
& \quad=\frac{4 \bar{h}^{2}}{\pi^{2}} \int_{0}^{s_{0}} e^{\beta\left(\theta-a_{0}\right)} v^{T}(\theta) R_{s} v(\theta) d \theta \geq 0 .
\end{aligned}
$$

Therefore, by adding (H.5) and (H.4), we obtain

$$
\begin{aligned}
& \int_{0}^{t} e^{\beta\left(\theta-a_{0}\right)}\left(\Delta_{s} v\right)^{T}(\theta) R_{s}\left(\Delta_{s} v\right)(\theta) d \theta \\
& \quad \leq \gamma_{s}^{2} \int_{0}^{t} e^{\beta\left(\theta-a_{0}\right)} \xi\left(v(\theta),\left(\Delta_{s} v\right)(\theta)\right) d \theta, \forall t \geq 0
\end{aligned}
$$

with $\gamma_{s}=\frac{2 \bar{h}}{\pi}$. Substituting $\xi\left(v(\theta),\left(\Delta_{s} v\right)(\theta)\right)$ from (H.3) in (H.6), we arrive at $\int_{0}^{t} \mathcal{S}_{s}\left(\theta, v(\theta),\left(\Delta_{s} v\right)(\theta)\right) d \theta \leq 0$, where $\mathcal{S}_{s}$ is given by $(32)$.

\section{Proof of Lemma 10}

From the definition of $\Delta_{d}$ in (28), we have that

$$
\left(\Delta_{d} w\right)(t)=\left\{\begin{array}{l}
0, \forall t \in\left[0, a_{0}\right), \\
0, \forall t \in\left[a_{k}, s_{k+1}\right), k \in \mathbb{N} \\
-\int_{s_{k-1}}^{s_{k}} w(\theta) d \theta, \forall t \in\left[s_{k}, a_{k}\right), k \in \mathbb{N}^{\star}
\end{array}\right.
$$

(1) For all $t \in\left[0, s_{1}\right)$ : We have $\left(\Delta_{d} w\right)(\theta)=0$ for all

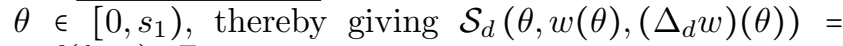
$-e^{\beta\left(\theta-a_{0}\right)} w^{T}(\theta) R_{d} w(\theta) d \theta \leq 0, \forall \theta \in\left[0, s_{1}\right)$, which implies $\int_{0}^{t} \mathcal{S}_{d}\left(\theta, w(\theta),\left(\Delta_{d} w\right)(\theta)\right) d \theta \leq 0, \forall t \in\left[0, s_{1}\right)$.
(2) For all $t \geq s_{1}$ : If $t \in\left[s_{k}, a_{k}\right)_{k \in \mathbb{N}^{*}}$, by virtue of Jensen's inequality, and using (I.1), we have that

$$
\begin{aligned}
e^{\beta\left(t-a_{0}\right)}\left(\Delta_{d} w\right)^{T}(t) R_{d} & \left(\Delta_{d} w\right)(t) \\
& \leq \bar{h} e^{\beta\left(t-a_{0}\right)} \int_{s_{k-1}}^{s_{k}} w^{T}(\theta) R_{d} w(\theta) d \theta
\end{aligned}
$$

as here we used that $s_{k}-s_{k+1} \leq \bar{h}, \forall k \in \mathbb{N}^{\star}$. Let $t \in$ $\left[s_{N}, s_{N+1}\right)_{N \in \mathbb{N}^{\star}}$, which implies that

$$
\begin{aligned}
& \int_{s_{1}}^{t} e^{\beta\left(\theta-a_{0}\right)}\left(\Delta_{d} w\right)^{T}(\theta) R_{d}\left(\Delta_{d} w\right)(\theta) d \theta \\
& =\sum_{k=1}^{N-1}\left(\int_{s_{k}}^{a_{k}} e^{\beta\left(\theta-a_{0}\right)}\left(\Delta_{d} w\right)^{T}(\theta) R_{d}\left(\Delta_{d} w\right)(\theta) d \theta\right. \\
& \left.+\int_{a_{k}}^{s_{k+1}} e^{\beta\left(\theta-a_{0}\right)}\left(\Delta_{d} w\right)^{T}(\theta) R_{d}\left(\Delta_{d} w\right)(\theta) d \theta\right) \\
& +\left\{\begin{array}{c}
\int_{s_{N}}^{t} e^{\beta\left(\theta-a_{0}\right)}\left(\Delta_{d} w\right)^{T}(\theta) R_{d}\left(\Delta_{d} w\right)(\theta) d \theta, t \in\left[s_{N}, a_{N}\right) \\
\int_{s_{N}}^{a_{N}} e^{\beta\left(\theta-a_{0}\right)}\left(\Delta_{d} w\right)^{T}(\theta) R_{d}\left(\Delta_{d} w\right)(\theta) d \theta \\
+\int_{a_{N}}^{t} e^{\beta\left(\theta-a_{0}\right)}\left(\Delta_{d} w\right)^{T}(\theta) R_{d}\left(\Delta_{d} w\right)(\theta) d \theta, \\
t \in\left[a_{N}, s_{N+1}\right) .
\end{array}\right.
\end{aligned}
$$

We know that for $t \in\left[a_{k}, s_{k+1}\right)_{k \in \mathbb{N}},\left(\Delta_{d} z\right)(t)=0$. Additionally, using the upper bound in (I.2), we have that

$$
\begin{aligned}
& \int_{s_{1}}^{t} e^{\beta\left(\theta-a_{0}\right)}\left(\Delta_{d} w\right)^{T}(\theta) R_{d}\left(\Delta_{d} w\right)(\theta) d \theta \\
& \leq \sum_{k=1}^{N-1}\left(\bar{h} \int_{s_{k}}^{a_{k}} e^{\beta\left(\theta-a_{0}\right)}\left(\int_{s_{k-1}}^{s_{k}} w^{T}(\eta) R_{d} w(\eta) d \eta\right) d \theta\right) \\
& +\left\{\begin{array}{c}
\bar{h} \int_{s_{N}}^{t} e^{\beta\left(\theta-a_{0}\right)}\left(\int_{s_{N-1}}^{s_{N}} w^{T}(\eta)\right. \\
\left.R_{d} w(\eta) d \eta\right) d \theta, t \in\left[s_{N}, a_{N}\right) \\
\bar{h} \int_{s_{N}}^{a_{N}} e^{\beta\left(\theta-a_{0}\right)}\left(\int_{s_{N-1}}^{s_{N}} w^{T}(\eta)\right. \\
\left.R_{d} w(\eta) d \eta\right) d \theta, t \in\left[a_{N}, s_{N+1}\right) .
\end{array}\right.
\end{aligned}
$$

Next, we simplify each of the integrals present in the right side of the inequality above. First, consider the term $\bar{h} \int_{s_{k}}^{a_{k}} e^{\beta\left(\theta-a_{0}\right)}\left(\int_{s_{k-1}}^{s_{k}} w^{T}(\eta) R_{d} w(\eta) d \eta\right) d \theta=$ $\bar{h} e^{-\beta a_{0}} \int_{s_{k}}^{a_{k}} e^{\beta \theta}\left(\int_{s_{k-1}}^{s_{k}} w^{T}(\eta) R_{d} w(\eta) d \eta\right) d \theta$. Let $\theta=s_{k}+s$ $\Rightarrow d \theta=d s$. This leads to

$$
\begin{aligned}
& \bar{h} \int_{s_{k}}^{a_{k}} e^{\beta\left(\theta-a_{0}\right)}\left(\int_{s_{k-1}}^{s_{k}} w^{T}(\eta) R_{d} w(\eta) d \eta\right) d \theta \\
& \quad \leq \bar{h} e^{-\beta a_{0}} \int_{0}^{\bar{\tau}} e^{\beta\left(s_{k}+s\right)}\left(\int_{s_{k-1}}^{s_{k}} w^{T}(\eta) R_{d} w(\eta) d \eta\right) d s .
\end{aligned}
$$

Since $s \in[0, \bar{\tau}]$ in (I.5), it can be stated that $e^{\beta\left(s_{k}+s\right)} \leq$ $e^{\beta\left(s_{k}+\bar{\tau}\right)}$. Hence,

$\bar{h} e^{-\beta a_{0}} \int_{0}^{\bar{\tau}} e^{\beta\left(s_{k}+s\right)}\left(\int_{s_{k-1}}^{s_{k}} w^{T}(\eta) R_{d} w(\eta) d \eta\right) d s$ $\leq \bar{h} e^{\beta\left(-a_{0}+\bar{\tau}\right)} \int_{0}^{\bar{\tau}} e^{\beta s_{k}}\left(\int_{s_{k-1}}^{s_{k}} w^{T}(\eta) R_{d} w(\eta) d \eta\right) d s$ $\leq \bar{h} e^{\beta\left(-a_{0}+\bar{\tau}\right)} \int_{0}^{\bar{\tau}}\left(\int_{s_{k-1}}^{s_{k}} e^{\beta s_{k}} w^{T}(\eta) R_{d} w(\eta) d \eta\right) d s$. Here, $\eta \in\left[s_{k-1}, s_{k}\right]$, which allows us to make the upper bounding $e^{\beta s_{k}} \leq e^{\beta(\eta+\bar{h})}$, thereby resulting in

$$
\begin{aligned}
\bar{h} & e^{\beta\left(-a_{0}+\bar{\tau}\right)} \int_{0}^{\bar{\tau}}\left(\int_{s_{k-1}}^{s_{k}} e^{\beta s_{k}} w^{T}(\eta) R_{d} w(\eta) d \eta\right) d s \\
& \leq \bar{h} e^{\beta(\bar{\tau}+\bar{h})} \int_{0}^{\bar{\tau}}\left(\int_{s_{k-1}}^{s_{k}} e^{\beta\left(\eta-a_{0}\right)} w^{T}(\eta) R_{d} w(\eta) d \eta\right) d s \\
& \leq \bar{h} \bar{\tau} e^{\beta(\bar{\tau}+\bar{h})} \int_{s_{k-1}}^{s_{k}} e^{\beta\left(\eta-a_{0}\right)} w^{T}(\eta) R_{d} w(\eta) d \eta .
\end{aligned}
$$


Thus, by combining (I.5)-(I.6), we have that $\bar{h} \int_{s_{k}}^{a_{k}} e^{\beta\left(\theta-a_{0}\right)}\left(\int_{s_{k-1}}^{s_{k}} w^{T}(\eta) R_{d} w(\eta) d \eta\right) d \theta$ $\leq \bar{h} \bar{\tau} e^{\beta(\bar{\tau}+\bar{h})} \int_{s_{k-1}}^{s_{k}} e^{\beta\left(\eta-a_{0}\right)} w^{T}(\eta) R_{d} w(\eta) d \eta$. Substituting this in (I.4) gives $\int_{s_{1}}^{t} e^{\beta\left(\theta-a_{0}\right)}\left(\Delta_{d} w\right)^{T}(\theta) R_{d}\left(\Delta_{d} w\right)(\theta) d \theta$ $\leq \quad \sum_{k=1}^{N-1}\left(\bar{h} \bar{\tau} e^{\beta(\bar{\tau}+\bar{h})} \int_{s_{k-1}}^{s_{k}} e^{\beta\left(\eta-a_{0}\right)} w^{T}(\eta) R_{d} w(\eta) d \eta\right)+$ $\bar{h} \bar{\tau} e^{\beta(\bar{\tau}+\bar{h})} \int_{s_{N-1}}^{s_{N}} e^{\beta\left(\eta-a_{0}\right)} w^{T}(\eta) R_{d} w(\eta) d \eta$ $\leq \bar{h} \bar{\tau} e^{\beta(\bar{\tau}+\bar{h})} \int_{s_{0}}^{s_{N}} e^{\beta\left(\eta-a_{0}\right)} w^{T}(\eta) R_{d} w(\eta) d \eta$. Therefore,

$$
\begin{aligned}
\int_{s_{1}}^{t} & e^{\beta\left(\theta-a_{0}\right)}\left(\Delta_{d} w\right)^{T}(\theta) R_{d}\left(\Delta_{d} w\right)(\theta) d \theta \\
& \leq \bar{h} \bar{\tau} e^{\beta(\bar{\tau}+\bar{h})} \int_{s_{0}}^{s_{N}} e^{\beta\left(\eta-a_{0}\right)} w^{T}(\eta) R_{d} w(\eta) d \eta
\end{aligned}
$$

Since $\left(\Delta_{d} w\right)(t)=0$ for $t<s_{1}$ (see $\left.(28)\right)$, we have that $\int_{s_{1}}^{t} e^{\beta\left(\theta-a_{0}\right)}\left(\Delta_{d} w\right)^{T}(\theta) R_{d}\left(\Delta_{d} w\right)(\theta) d \theta$

$=\int_{0}^{t} e^{\beta\left(\theta-a_{0}\right)}\left(\Delta_{d} w\right)^{T}(\theta) R_{d}\left(\Delta_{d} w\right)(\theta) d \theta$. Additionally, since $w \in \mathcal{W}^{m_{p}}$, we can state $e^{\beta\left(\eta-a_{0}\right)} w^{T}(\eta) R_{d} w(\eta) \geq$ $0, \forall \eta \geq 0$, thereby implying that

$\bar{h} \bar{\tau} e^{\beta(\bar{\tau}+\bar{h})} \int_{s_{0}}^{s_{N}} e^{\beta\left(\eta-a_{0}\right)} w^{T}(\eta) R_{d} w(\eta) d \eta$

$\leq \bar{h} \bar{\tau} e^{\beta(\bar{\tau}+\bar{h})} \int_{0}^{t} e^{\beta\left(\eta-a_{0}\right)} w^{T}(\eta) R_{d} w(\eta) d \eta$. Consequently, we can rewrite (I.7) as

$\int_{0}^{t} e^{\beta\left(\theta-a_{0}\right)}\left(\Delta_{d} w\right)^{T}(\theta) R_{d}\left(\Delta_{d} w\right)(\theta) d \theta$

$\leq \bar{h} \bar{\tau} e^{\beta(\bar{\tau}+\bar{h})} \int_{0}^{t} e^{\beta\left(\eta-a_{0}\right)} w^{T}(\eta) R_{d} w(\eta) d \eta$. By rearranging the terms, we have $\int_{0}^{t} \mathcal{S}_{d}\left(\theta, w(\theta),\left(\Delta_{d} w\right)(\theta)\right) d \theta \leq$ $0, \quad \forall t \geq 0$, where $\mathcal{S}_{d}\left(\theta, w(\theta),\left(\Delta_{d} w\right)(\theta)\right)$ is given by (34).

\section{J Proof of Theorem 12}

Let us recall the linear sampled-data system $\mathcal{P}_{L}$ described in Section 4.3 by (20). The sampling-induced error is given by

$e_{s}(t)=\left\{\begin{array}{l}0, \forall t \in\left[0, s_{0}\right), \\ K x\left(s_{k}\right)-K x(t), \forall t \in\left[s_{k}, s_{k+1}\right), k \in \mathbb{N},\end{array}\right.$

$=\left(\Delta_{s}(K \dot{x})\right)(t)$, where $\Delta_{s}$ is given by $(27)$. Similarly, the delay-induced error is given by

$$
e_{d}(t)=\left\{\begin{array}{l}
0, \forall t \in\left[0, a_{0}\right), \\
0, \forall t \in\left[a_{k-1}, s_{k}\right), k \in \mathbb{N}^{\star}, \\
K x\left(s_{k-1}\right)-K x\left(s_{k}\right), \forall t \in\left[s_{k}, a_{k}\right), k \in \mathbb{N}^{\star},
\end{array}\right.
$$

$=\left(\Delta_{d}(K \dot{x})\right)(t)$, where $\Delta_{d}$ is given by (28). Additionally, the functions defined in (30) are given by

$$
\begin{aligned}
& \bar{f}_{0}(x(t))=A x(t), \bar{h}_{0}(x(t))=\left[\begin{array}{l}
K A x(t) \\
K A x(t)
\end{array}\right], \\
& \bar{f}(x(t))=\bar{A} x(t), \bar{g}(x(t))=\left[\begin{array}{cc}
B & B
\end{array}\right], \\
& \bar{h}(x(t))=\left[\begin{array}{l}
K \bar{A} x(t) \\
K \bar{A} x(t)
\end{array}\right], \bar{l}(x(t))=\left[\begin{array}{l}
K B \\
K B
\end{array}\right] .
\end{aligned}
$$

Let us consider that condition (35) holds. Then, we proceed to prove that the assumptions introduced in Theorem 1 will hold for the storage function $V(x)=x^{T} P x$ and the supply function $\mathcal{S}: \mathbb{R}^{+} \times \mathbb{R}^{2 n} \times \mathbb{R}^{2 n} \rightarrow \mathbb{R}$ given by

$$
\begin{array}{r}
\mathcal{S}(t, y(t), \omega(t))=\mathcal{S}_{s}\left(t,\left[\begin{array}{ll}
I & 0
\end{array}\right] y(t),\left[\begin{array}{ll}
I & 0
\end{array}\right] \omega(t)\right) \\
+\mathcal{S}_{d}\left(t,\left[\begin{array}{ll}
0 & I
\end{array}\right] y(t),\left[\begin{array}{ll}
0 & I
\end{array}\right] \omega(t)\right) \\
=\mathcal{S}_{s}\left(t, y_{1}(t), e_{s}(t)\right)+\mathcal{S}_{d}\left(t, y_{2}(t), e_{d}(t)\right)
\end{array}
$$

where $\mathcal{S}_{s}$ and $\mathcal{S}_{d}$ are defined by (32) and (34), respectively, with $\beta=\alpha$. Additionally, based on the functions given in (J.1), we have $y_{1}(t)=y_{2}(t)=K \dot{x}(t)$. Let us now show that the assumptions in Theorem 1 are validated. (1) Satisfying Assumption 1, i.e., (9): By virtue of Lemmas 9 and 10, we have that

$\int_{0}^{t} \mathcal{S}_{s}\left(\theta, y_{1}(\theta),\left(\Delta_{s} y_{1}\right)(\theta)\right) d \theta \leq 0, \quad \forall t \geq 0$, and $\int_{0}^{t} \mathcal{S}_{d}\left(\theta, y_{2}(\theta),\left(\Delta_{d} y_{2}\right)(\theta)\right) d \theta \leq 0, \quad \forall t \geq 0$. Consequently, as per the definition of the supply function in (J.2), we obtain $\int_{0}^{t} \mathcal{S}(\theta, y(\theta), \omega(\theta)) d \theta \leq 0, \forall t \geq 0$.

(2) Satisfying Assumption 2, i.e., (10): With $V(x)=$

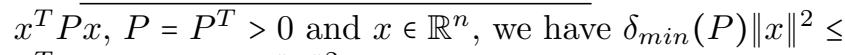
$x^{T} P x \leq \delta_{\max }(P)\|x\|^{2}$, implying Assumption 2 is satisfied with $q=2, c_{1}=\delta_{\min }(P)$ and $c_{2}=\delta_{\max }(P)$.

(3) Satisfying Assumption 3, inequality (11): Consider the function $\mathcal{S}(t, y(t), \omega(t))$ given in (J.2). Then, we need to prove that $-\mathcal{S}(t, y(t), \omega(t)) \leq \rho V(x(t)), \forall t \in$ $\left[0, a_{0}\right)$. We proceed to prove this inequality by considering the time intervals $\left[0, s_{0}\right)$ and $\left[s_{0}, a_{0}\right)$ separately.

For all $t \in\left[0, s_{0}\right)$ : Using the definition of system $\Sigma$ in $(\overline{7}),(\overline{30})$, and the operator $\Delta$ defined in (8), (28) we have that $y(t)=\bar{h}_{0}(x(t))=\left[\begin{array}{l}K A x(t) \\ K A x(t)\end{array}\right]$, and $\omega(t)=\left[\begin{array}{l}e_{s}(t) \\ e_{d}(t)\end{array}\right]=\left[\begin{array}{l}0 \\ 0\end{array}\right], \forall t \in\left[0, s_{0}\right)$. Hence, for all $t \in\left[0, s_{0}\right),-\mathcal{S}(t, y(t), \omega(t))=-\mathcal{S}\left(t, \bar{h}_{0}(x(t)), 0\right)=$ $-\left(\mathcal{S}_{s}(t, K A x(t), 0)+\mathcal{S}_{d}(t, K A x(t), 0)\right)$

$$
=e^{\alpha\left(t-a_{0}\right)}\left(x^{T}(t)(K A)^{T}\left[\gamma_{s}^{2} R_{s}+\gamma_{d} R_{d}\right](K A) x(t)\right) \text {. }
$$

Therefore,

$$
-\mathcal{S}(t, y(t), \omega(t)) \leq \rho_{1} V(x(t))
$$

with

$$
\rho_{1}=\frac{e^{-\alpha \tau_{0}} \delta_{\max }\left[(K A)^{T}\left[\gamma_{s}^{2} R_{s}+\gamma_{d} R_{d}\right](K A)\right]}{\delta_{\min }(P)},
$$

where $\gamma_{s}=\frac{2 \bar{h}}{\pi}$ and $\gamma_{d}=\bar{h} \bar{\tau} e^{\alpha(\bar{h}+\bar{\tau})}$.

For all $t \in\left[s_{0}, a_{0}\right):$ From (J.1), we have $y(t)=\left[\begin{array}{l}y_{1}(t) \\ y_{2}(t)\end{array}\right]$ with

$$
y_{1}(t)=y_{2}(t)=K A x(t)
$$

and $\omega(t)=\left[\begin{array}{l}e_{s}(t) \\ e_{d}(t)\end{array}\right]=\left[\begin{array}{c}K x\left(s_{0}\right)-K x(t) \\ 0\end{array}\right]$. Since the system is in open loop for all $t \in\left[s_{0}, a_{0}\right), x\left(s_{0}\right)=$ 
$e^{A\left(s_{0}-t\right)} x(t)$. Therefore, we have that

$$
\left[\begin{array}{l}
e_{s}(t) \\
e_{d}(t)
\end{array}\right]=\left[\begin{array}{c}
K\left[e^{A\left(s_{0}-t\right)}-I\right] x(t) \\
0
\end{array}\right], \forall t \in\left[s_{0}, a_{0}\right) .
$$

Now, consider the function $\mathcal{S}_{s}$ defined in (32). Since we have already shown in Lemma 7 that $\left(\Delta_{s} y_{1}\right)(t)=e_{s}(t)$, we have that

$$
\begin{aligned}
& \mathcal{S}_{s}\left(t, y_{1}(t),\left(\Delta_{s} y_{1}\right)(t)\right)=\mathcal{S}_{s}\left(t, y_{1}(t), e_{s}(t)\right) \\
& \quad=e^{\alpha\left(t-a_{0}\right)}\left[\begin{array}{l}
y_{1}(t) \\
e_{s}(t)
\end{array}\right]^{T}\left[\begin{array}{cc}
-\gamma_{s}^{2} R_{s} & \gamma_{s}^{2} \frac{\alpha}{2} R_{s} \\
\gamma_{s}^{2} \frac{\alpha}{2} R_{s} & \left(1-\gamma_{s}^{2} \frac{\alpha^{2}}{4}\right) R_{s}
\end{array}\right]\left[\begin{array}{l}
y_{1}(t) \\
e_{s}(t)
\end{array}\right],
\end{aligned}
$$

and thus, from (J.5) and (J.6), we get $\mathcal{S}_{s}\left(t, y_{1}(t), e_{s}(t)\right)=$ $\mathcal{S}_{s}\left(t, K A x(t), K\left[e^{A\left(s_{0}-t\right)}-I\right] x(t)\right)=x^{T}(t) \mathcal{M}(t) x(t)$, $\forall t \in\left[s_{0}, a_{0}\right)$, where $\mathcal{M}(t)=e^{\alpha\left(t-a_{0}\right)}\left[\begin{array}{c}K A \\ K\left[e^{A\left(s_{0}-t\right)}-I\right]\end{array}\right]^{T}$ $\left[\begin{array}{cc}-\gamma_{s}^{2} R_{s} & \gamma_{s}^{2} \frac{\alpha}{2} R_{s} \\ \gamma_{s}^{2} \frac{\alpha}{2} R_{s} & \left(1-\gamma_{s}^{2} \frac{\alpha^{2}}{4}\right) R_{s}\end{array}\right]\left[\begin{array}{c}K A \\ K\left[e^{A\left(s_{0}-t\right)}-I\right]\end{array}\right] . \quad$ Similarly, considering the function $\mathcal{S}_{d}$ defined by $(34)$, we have that

$$
\begin{aligned}
& \mathcal{S}_{d}\left(t, y_{2}(t),\left(\Delta_{d} y_{2}\right)(t)\right)=\mathcal{S}_{d}\left(t, y_{2}(t), e_{d}(t)\right) \\
& =e^{\alpha\left(t-a_{0}\right)}\left[\begin{array}{l}
y_{2}(t) \\
e_{d}(t)
\end{array}\right]^{T}\left[\begin{array}{rr}
-\gamma_{d} R_{d} & 0 \\
0 & R_{d}
\end{array}\right]\left[\begin{array}{l}
y_{2}(t) \\
e_{d}(t)
\end{array}\right],
\end{aligned}
$$

and thus, from (J.5) and (J.6), $\mathcal{S}_{d}\left(t, y_{2}(t), e_{d}(t)\right)=$ $\mathcal{S}_{d}(t, K A x(t), 0)=x^{T}(t) \mathcal{N}(t) x(t), \forall t \in\left[s_{0}, a_{0}\right)$, with $\mathcal{N}(t)=-\gamma_{d} e^{\alpha\left(t-a_{0}\right)}(K A)^{T} R_{d}(K A)$. Therefore, we have the total supply function $\mathcal{S}$ satisfying $-\mathcal{S}(t, y(t), \omega(t))=$ $-\mathcal{S}_{s}\left(t, y_{1}(t), e_{s}(t)\right)-\mathcal{S}_{d}\left(t, y_{2}(t), e_{d}(t)\right)=x^{T}(t) M(t) x(t)$, where $M(t)=-\mathcal{M}(t)-\mathcal{N}(t)$. Hence, for all $t \in\left[s_{0}, a_{0}\right)$, we can state that

$$
-\mathcal{S}(t, y(t), \omega(t)) \leq \rho_{2} V(x(t))
$$

where

$$
\rho_{2}=\frac{\max _{\theta \in\left[s_{0}, a_{0}\right]}\left\{\delta_{\max }[M(\theta)]\right\}}{\delta_{\min }(P)} .
$$

Then, from (J.3) and (J.9), we have $-\mathcal{S}(t, y(t), \omega(t)) \leq$ $\rho V(x(t)), \forall t \in\left[0, a_{0}\right)$, where $\rho=\max \left\{\rho_{1}, \rho_{2}\right\}$ with $\rho_{1}$ and $\rho_{2}$ given by (J.4) and (J.10), respectively.

(4) Satisfying Assumption 3, inequality (12): We have

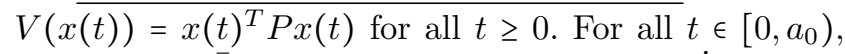
since $\dot{x}(t)=\bar{f}_{0}(x(t))=A x(t)$, it holds that $\dot{V}(x(t))=$ $x(t)^{T}\left[A^{T} P+P A\right] x(t) \geq \frac{\delta_{\min }\left(A^{T} P+P A\right)}{\delta_{\max }(P)} V(x(t))$. Therefore, it is clear that inequality (12) is satisfied for any $\lambda \leq \frac{\delta_{\min }\left(A^{T} P+P A\right)}{\delta_{\max }(P)}$.

(5) Satisfying Assumption 3, inequality (13):
Consider the function $W(t)=\dot{V}(x(t))+\alpha V(x(t))-$ $e^{-\alpha\left(t-a_{0}\right)} \mathcal{S}(t, y(t), e(t))$, defined for all $t \geq a_{0}$ with $V(x)=x^{T} P x$, and the function $\mathcal{S}$ defined by (J.2). Clearly, the inequality in (13) holds if $W(t) \leq 0$, for all $t \geq a_{0}$. Using the definitions of $\mathcal{S}_{s}\left(t, y_{1}(t), e_{s}(t)\right)$ and $\mathcal{S}_{d}\left(t, y_{2}(t), e_{d}(t)\right)$ in (J.7) and (J.8), respectively, and from (J.1), since $y_{1}(t)=y_{2}(t)=K \dot{x}(t)$, for all $t \geq 0$, we have that

$$
\mathcal{S}(t, y(t), \omega(t))=e^{\alpha\left(t-a_{0}\right)}\left[\begin{array}{c}
K \dot{x}(t) \\
e_{s}(t) \\
e_{d}(t)
\end{array}\right]^{T} \Psi\left[\begin{array}{c}
K \dot{x}(t) \\
e_{s}(t) \\
e_{d}(t)
\end{array}\right],
$$

with, $\Psi=\left[\begin{array}{ccc}-\gamma_{s}^{2} R_{s}-\gamma_{d} R_{d} & \gamma_{s}^{2} \frac{\alpha}{2} R_{s} & 0 \\ \gamma_{s}^{2} \frac{\alpha}{2} R_{s} & \left(1-\gamma_{s}^{2} \frac{\alpha^{2}}{4}\right) R_{s} & 0 \\ 0 & 0 & R_{d}\end{array}\right]$. From the system dynamics defined by (7) and (J.1), we have that $\left[\begin{array}{c}K \dot{x}(t) \\ e_{s}(t) \\ e_{d}(t)\end{array}\right]=\left[\begin{array}{ccc}K \bar{A} & K B & K B \\ 0 & I & 0 \\ 0 & 0 & I\end{array}\right]\left[\begin{array}{l}x(t) \\ e_{s}(t) \\ e_{d}(t)\end{array}\right]=\left[\begin{array}{c:c}K \bar{A} \backslash K \bar{B} \\ \hdashline 0 & I\end{array}\right]\left[\begin{array}{c}x(t) \\ \hdashline e_{s}(t) \\ e_{d}(t)\end{array}\right], \quad$ with $\bar{A}=A+B K$ and $\bar{B}=\left[\begin{array}{ll}B & B\end{array}\right]$. Therefore, from (J.11), we have that $\mathcal{S}(t, y(t), \omega(t))=e^{\alpha\left(t-a_{0}\right)}\left[\begin{array}{c}x(t) \\ e_{s}(t) \\ e_{d}(t)\end{array}\right]^{T} N\left[\begin{array}{c}x(t) \\ e_{s}(t) \\ e_{d}(t)\end{array}\right]$, where $N=\left[\begin{array}{cc}K \bar{A} & K \bar{B} \\ 0 & I\end{array}\right]^{T} \Psi\left[\begin{array}{cc}K \bar{A} & K \bar{B} \\ 0 & I\end{array}\right]$. Therefore, $\forall t \geq$ $a_{0}$, we have that $W(t)=\dot{V}(x(t))+\alpha V(x(t))-$ $e^{-\alpha\left(t-a_{0}\right)} \mathcal{S}(t, y(t), e(t))=\left[\begin{array}{c}x(t) \\ e_{s}(t) \\ e_{d}(t)\end{array}\right]^{T}\left\{\left[\begin{array}{c:cc}\bar{A}^{T} P+P \bar{A} \backslash P B & P B \\ \hdashline B^{T} P & 0 & 0 \\ B^{T} P & 0 & 0\end{array}\right]+\right.$ $\left.\alpha\left[\begin{array}{lll}P & 0 & 0 \\ 0 & 0 & 0 \\ 0 & 0 & 0\end{array}\right]-N\right\}\left[\begin{array}{c}x(t) \\ e_{s}(t) \\ e_{d}(t)\end{array}\right]=\left[\begin{array}{c}x(t) \\ e_{s}(t) \\ e_{d}(t)\end{array}\right]^{T} \Gamma\left[\begin{array}{c}x(t) \\ e_{s}(t) \\ e_{d}(t)\end{array}\right]$,

with $\Gamma=\left[\begin{array}{cc}\bar{A}^{T} P+P \bar{A}+\alpha P & P \bar{B} \\ \bar{B}^{T} P & 0\end{array}\right]+\left[\begin{array}{cc}K \bar{A} & K \bar{B} \\ 0 & I\end{array}\right]^{T} \Phi\left[\begin{array}{cc}K \bar{A} & K \bar{B} \\ 0 & I\end{array}\right]$, with $\bar{A}=A+B K, \bar{B}=[B B]$, and $\Phi=-\Psi$ described in (36). A sufficient condition for $W(t) \leq 0, \forall t \geq a_{0}$ is $\Gamma \leq 0$, and guaranteed by (35). Consequently, we have proved that the inequality (13) is satisfied.

We have shown that all the assumptions of Theorem 1 hold for $V(x)=x^{T} P x$ and $\mathcal{S}(t, y(t), \omega(t))$ defined by (J.2) and hence, using Theorem 1 , system $\mathcal{P}$ is exponentially stable with a decay rate greater than or equal to $\alpha / 2$. 